\title{
Multimodal Processes Rescheduling: Cyclic Steady States Space Approach
}

\author{
Grzegorz Bocewicz, ${ }^{1}$ Robert Wójcik, ${ }^{2}$ Zbigniew Antoni Banaszak, ${ }^{3}$ and Paweł Pawlewski ${ }^{4}$ \\ ${ }^{1}$ Department of Electronics and Computer Science, Koszalin University of Technology, Koszalin, Poland \\ ${ }^{2}$ Institute of Computer Engineering, Control and Robotics, Wrocław University of Technology, Wrocław, Poland \\ ${ }^{3}$ Department of Business Informatics, Warsaw University of Technology, Warsaw, Poland \\ ${ }^{4}$ Faculty of Engineering Management, Poznań University of Technology, Poznań, Poland
}

Correspondence should be addressed to Grzegorz Bocewicz; bocewicz@ie.tu.koszalin.pl

Received 29 March 2013; Accepted 22 September 2013

Academic Editor: Zhichun Yang

Copyright (C) 2013 Grzegorz Bocewicz et al. This is an open access article distributed under the Creative Commons Attribution License, which permits unrestricted use, distribution, and reproduction in any medium, provided the original work is properly cited.

\begin{abstract}
The paper concerns cyclic scheduling problems arising in a Multimodal Transportation Network (MTN) in which several unimodal networks (AGVs, hoists, lifts, etc.) interact with each other via common shared workstations as to provide a variety of demandresponsive material handling operations. The material handling transport modes provide movement of work-pieces between workstations along their manufacturing routes in the MTN. The goal is to provide a declarative framework enabling to state a constraint satisfaction problem aimed at AGVs fleet match-up scheduling taking into consideration assumed itineraries of concurrently manufactured product types. In that context, treating the different product types as a set of cyclic multimodal processes is the main objective to discuss the conditions sufficient for FMS rescheduling imposed by production orders changes. To conclude, the conditions sufficient for an FMS rescheduling imposed by changes of production orders treated as cyclic multimodal processes are stated as the paper's main contribution.
\end{abstract}

\section{Introduction}

The design and operational issues arising in an Automated Guided Vehicle (AGV) served Flexible Manufacturing System (FMS) when deciding on how to achieve a desired system performance are usually hard to determine and evaluate. This is because productivity of an AGV-served flow shop producing a set of different kinds of products, repetitively, depends on both the job flow sequencing (aimed at maximizing throughput or minimizing cycle time) and the material handling system required to achieve a prespecified throughput, that is, AGVs fleet sizing, assignment and scheduling [1-5]. So, assuming a deterministic system where demand is known in advance and the processing times of each job on each machine is a known constant, in order to improve its productivity, the AGV fleet size and the scheduling/dispatching influence on the system throughput are usually examined $[1,6-9]$.
In opposite to conventionally applied approach assuming AGVs fleet sizing, routing, and timetabling while matching up prescheduled multiproduct manufacturing flow (i.e., providing detailed transportation route and materials load/ unload schedule between workstations in FMS), we propose AGVs following a Multimodal Transportation Network (MTN) concept. MTN can be seen as composition of several unimodal networks (AGVs, hoists, lifts, etc.) interacting with each other via common shared workstations as to provide a variety of demand-responsive material handling operations. The material handling transport modes provide movement of work-pieces between workstations along their manufacturing routes in the MTN. The MTN encompasses a well-known multimodal approach to freight transportation supply chains and passenger transportation systems. So, similarly to such systems having ability to use the appropriate mode of transport for movement of goods and people, the assumed material 
handling transport modes provide movement of work-pieces between workstations along their manufacturing routes in the MTN. In that context, assuming that the modes can be treated as cyclic local transportation processes, and material (e.g., work-pieces/tools) flows supported by them can be treated as multimodal processes, the problems considered can be seen either as aimed at designing of a multimodal network infrastructure guaranteeing assumed materials flow or focused on work-pieces routing ensuring lag-free workstations service.

The problems arising concerning material transportation routing and scheduling belong to NP-hard ones [2]. Since the steady state of production flows has a cyclic character, hence servicing them AGV-served transportation processes (usually executed along loop-like routes) encompasses also cyclic behavior. That means that the periodicity of FMS [10] depends on both the periodicity of production flow cyclic schedule and following this schedule the AGVs periodicity. Of course, the Multimodal Transportation Network (MTN) throughput is maximized by the minimization of its cycle time.

It seems to be obvious that not all the behaviors (including cyclic ones) are reachable under constraints imposed by the system's structure. The similar observation concerns the system's behavior that can be achieved in systems possessing specific structural constraints. Since system constraints determine its behavior, both the system structure and the desired cyclic schedule have to be considered simultaneously. So, the problem solution requires that the system structure must be determined for the purpose of processes scheduling, yet scheduling must be done to devise the system configuration. In that context, our contribution provides a discussion of some solvability issues concerning cyclic processes dispatching problems, especially the conditions guaranteeing solvability of the cyclic processes scheduling as well as direct and/or indirect transitions between assumed cyclic steady states. Their examination may replace exhaustive searches for solution satisfying required system capabilities.

Many models and methods have been considered to date [11]. Among them, the mathematical programming approach $[1,12]$, max-plus algebra [13], constraint logic programming [14-16] evolutionary algorithms, Petri nets [17, 18], and heuristic frameworks [19] belong to the more frequently used. Most of these are oriented at finding a minimal cycle or maximal throughput while assuming deadlock-free processes flow. Note that processes' operations are blocking if they must stay on a resource (e.g., the station, the machine) after finishing when the next resource is occupied by a job from another process. During this stay the resource is blocked for other processes. The approaches trying to estimate the cycle time from cyclic processes structure and the synchronization mechanism employed (i.e., mutual exclusion instances) while taking into account deadlock phenomena are quite unique.

In our approach a declarative framework aimed at refinement and prototyping of the cyclic steady states for concurrently executed cyclic processes modelling material handling systems is employed. These systems are of the type of AGVs fleets in the flexible manufacturing systems (FMS) and are frequently encountered in industry. The following questions are the main focus of the research. Can the assumed material handling system, for example, AGVs, meet load/unload deadlines imposed by flow of scheduled work-pieces processing? Does there exist sufficient AGVS enabling to schedule the AGVs fleet as to ensure lag-free service of scheduled work pieces processing? So, the main question is Can the assumed AGVs fleet assignment reach its goal subject to constraints assumed on concurrent multiproduct manufacturing at hand?

Moreover the declarative framework enables to state a problem of multimodal processes rescheduling which boils down to searching for transient periods allowing the mutual reachability of MTN cyclic behaviors. In the case of MTNs, distinguishing many different cyclic steady states (cyclic behaviors), the following questions play a pivotal role. Does there exist the smooth (direct) transition between two assumed cyclic behaviors? What conditions guarantee the reachability of a given cyclic behavior from any other ones? Is it possible to come back to a given cyclic behavior from any disturbance-born one, for instance, caused by operation time delays, damage of devices, route modifications, and so forth?

In other words, the paper's objective concerns the MTN infrastructure assessment from the perspective of possible FMS oriented requirements imposed by AGVs fleet assignment, sizing, and scheduling (problems of cyclic processes scheduling and rescheduling). Due to the complexity implied in answering the above questions, the combined problem remains unsolved for all practical purposes. This is especially problematic as many manufacturing companies would stand to reap considerable rewards through better fleet assignment and scheduling. The presented approach addresses this through solving the combined rather than the separate problems individually.

The rest of the paper is organized as follows. Section 2 introduces the Automated Guided Vehicles System (AGVS) modelled in terms of concurrently flowing cyclic processes (SCCPs) and a cyclic steady state space concept. Section 3 regarding multimodal processes rescheduling provides a problem statement concerning AGVs fleet match-up scheduling with an assumed multiproduct manufacturing flow schedule. Sufficient conditions allowing one to search for states with mutual allocation as well as illustrative example of implementing them algorithm usage are discussed. The related work and concluding remarks are presented in Sections 4 and 5, respectively.

\section{Multimodal Network Modeling}

Considered case of multimodal processes rescheduling problem is presented on the example of Automated Guided Vehicle Systems (AGVS) modeled by Systems of Concurrently Flowing Cyclic Processes (SCCPs). To solve this kind of problems a nonempty state space, that is, containing nonempty set of possible cyclic behaviors, is required. For that reason the conditions guaranteeing cyclic behavior are presented, primarily. They are specified in terms of a declarative model formalism distinguishing the structure and behavior as the 
main components of an SCCP. Relationship linking the structure and reachable behaviors is the main subject of this section.

2.1. Model of AGVS. Automated Guided Vehicle Systems are used for material handling within a Flexible Manufacturing System (FMS) and provide asynchronous movement pallets of products through a network of guide paths between the workstations by the AGVs. Each workstation is connected to the guide path network by a pick-up/delivery station where pallets are transferred from/to the AGVs.

In AGVS literature, most are related to AGVS design issues, which include determination of the number of vehicles required, flow path design, and route planning as well as vehicle dispatching and traffic management. Recently, an integrated problem of dispatching and conflict free routing of AGVs, that is, integrating the simultaneous assignment, scheduling, and conflict free routing of the vehicles, is receiving increasing attention. The above mentioned problems, in the general case, belong to the class of NP-hard problems. Since most processes observed in steady state manufacturing are periodic, cyclic schedules and following them, cyclic scheduling methods can be considered. Since cyclic schedules encompass repetitive character of manufacturing processes, the cyclic processes modeling approach seems to be a reasonable perspective [13-15].

To present some of the design and operational issues that arise in repetitive manufacturing systems served by AGVs in network of loops layout with unidirectional material flow, a case example of a simple FMS is shown in Figure 1. The concurrent multiproduct flows are depicted by bold green, blue, and orange color lines, while the transportation loop-like networks are distinguished by double solid line. Both kinds of material (jobs) and transportation (AGVs) flows shown in Figure 1 can be modeled in terms of Systems of Concurrently Flowing Cyclic Processes $[14,15]$ as shown in Figure 2. In turn, the SCCP framework provides a formal model enabling to state and resolve problems of AGV fleet size minimization as well as steady state cycle time minimization. The cycle time minimization is required to obtain maximum throughput rate.

Eight local cyclic processes are considered, namely, $P_{1}$, $P_{2}, P_{3}, P_{4}, P_{5}, P_{6}, P_{7}$, and $P_{8}$. The processes follow the routes are composed of transportation sectors and machines (distinguished in Figure 2 by the set of resources $R=$ $\left\{R_{1}, \ldots, R_{c}, \ldots, R_{33}\right\}, R_{c}$ - the $c$ th resource). Some of the local cyclic processes are pipeline flow processes; that is, they contain streams (representing vehicles from Figure 1) of the processes following the same route while occupying different resources (sectors). For instance, processes $P_{4}, P_{5}$, and $P_{6}$ contain two streams: $P_{4}=\left\{P_{4}^{1}, P_{4}^{2}\right\}, P_{5}=\left\{P_{5}^{1}, P_{5}^{2}\right\}$, and $P_{6}=$ $\left\{P_{6}^{1}, P_{6}^{2}\right\}$, respectively, and $P_{2}$ contains three streams: $P_{2}=$ $\left\{P_{2}^{1}, P_{2}^{2}, P_{2}^{3}\right\}$, that is, the processes (vehicles) moving along the same route. The remaining local processes contain unique streams: $P_{1}=\left\{P_{1}^{1}\right\}, P_{3}=\left\{P_{3}^{1}\right\}, P_{7}=\left\{P_{7}^{1}\right\}$, and $P_{8}=\left\{P_{8}^{1}\right\}$. In other words, the streams $P_{1}^{1}, P_{2}^{1}, P_{2}^{2}, P_{2}^{3}, P_{3}^{1}, P_{4}^{1}, P_{4}^{2}, P_{5}^{1}, P_{5}^{2}$, $P_{6}^{1}, P_{6}^{2}, P_{7}^{1}$, and $P_{8}^{1}$ represent the 13 vehicles from Figure 1 . The $k$ th stream of the $i$ th local process $P_{i}$ is denoted by $P_{i}^{k}$.
Apart from local processes, we consider three multimodal processes (i.e., processes executed along the routes consisting of parts of the routes of local processes): $m P_{1}, m P_{2}$, and $m P_{3}$.

For example, the production route depicted by the orange line corresponds to the multimodal process $m P_{1}$ supported by AGVs (arbitrarily given), which in turn encompass local transportation streams $P_{1}^{1}, P_{2}^{1}, P_{2}^{3}$, and $P_{4}^{2}$. This means that the production route specifying how a multimodal process is executed can be considered to be composed of parts of the routes of local cyclic processes.

In the system considered each multimodal process consists of four streams: $m P_{i}=\left\{m P_{i}^{1}, m P_{i}^{2}, m P_{i}^{3}, m P_{i}^{4}\right\}, i=$ $1,2,3$, which means that along each production route four work- pieces are processed serially (four balls on the one production lines in Figure 1).

Processes can interact with each other through shared resources, that is, the transportation sectors. The routes of the considered local processes (streams) are as follows (see Figure 2):

$$
\begin{gathered}
p_{1}^{1}=\left(R_{16}, R_{3}, R_{13}, R_{6}\right), \\
p_{2}^{1}=\left(R_{21}, R_{8}, R_{18}, R_{9}, R_{14}, R_{6}, R_{17}, R_{5}\right), \\
p_{2}^{2}=\left(R_{17}, R_{5}, R_{21}, R_{8}, R_{18}, R_{9}, R_{14}, R_{6}\right), \\
p_{2}^{3}=\left(R_{14}, R_{6}, R_{17}, R_{5}, R_{21}, R_{8}, R_{18}, R_{9}\right), \\
p_{4}^{1}=\left(R_{29}^{1}, R_{7}, R_{25}, R_{8}, R_{22}, R_{11}, R_{26}, R_{10}\right), \\
p_{4}^{2}=\left(R_{26}, R_{10}, R_{29}, R_{7}, R_{25}, R_{8}, R_{22}, R_{11}\right), \\
p_{5}^{1}=\left(R_{2}, R_{20}, R_{5}, R_{24}, R_{4}, R_{27}, R_{1}, R_{23}\right), \\
p_{5}^{2}=\left(R_{27}, R_{1}, R_{23}, R_{2}, R_{20}, R_{5}, R_{24}, R_{4}\right), \\
p_{6}^{1}=\left(R_{32}, R_{4}, R_{28}, R_{7}, R_{31}\right), \\
p_{8}^{2}=\left(R_{31}, R_{32}, R_{4}, R_{28}, R_{7}\right), \\
\left.p_{33}^{1}=\left(R_{1}, R_{7}, R_{29}, R_{10}\right), R_{4}, R_{30}\right),
\end{gathered}
$$

where $R_{1}-R_{2}, R_{4}-R_{11}, R_{14}, R_{17}-R_{18}, R_{20}-R_{29}, R_{31}$, and $R_{32}$ (due to two streams of process $P_{6}$, resources $R_{31}, R_{32}$ represent one sector) are resources shared by local processes, that is, by at least two streams, and $R_{3}, R_{12}, R_{13}, R_{15}, R_{16}, R_{19}, R_{30}$, and $R_{33}$ are the nonshared resources, that is, exclusively used by one stream only.

In the general case, the route $p_{i}^{k}$ is the sequence of resources used in order to execute the operations of the stream $P_{i}^{k}$. Note that the streams $P_{2}^{1}, P_{2}^{2}$, and $P_{2}^{3}$ which belong to $P_{2}$ and the streams of processes $P_{4}, P_{5}$, and $P_{6}$ follow the same route but start from different resources (these streams correspond to vehicles moving along the same route). 

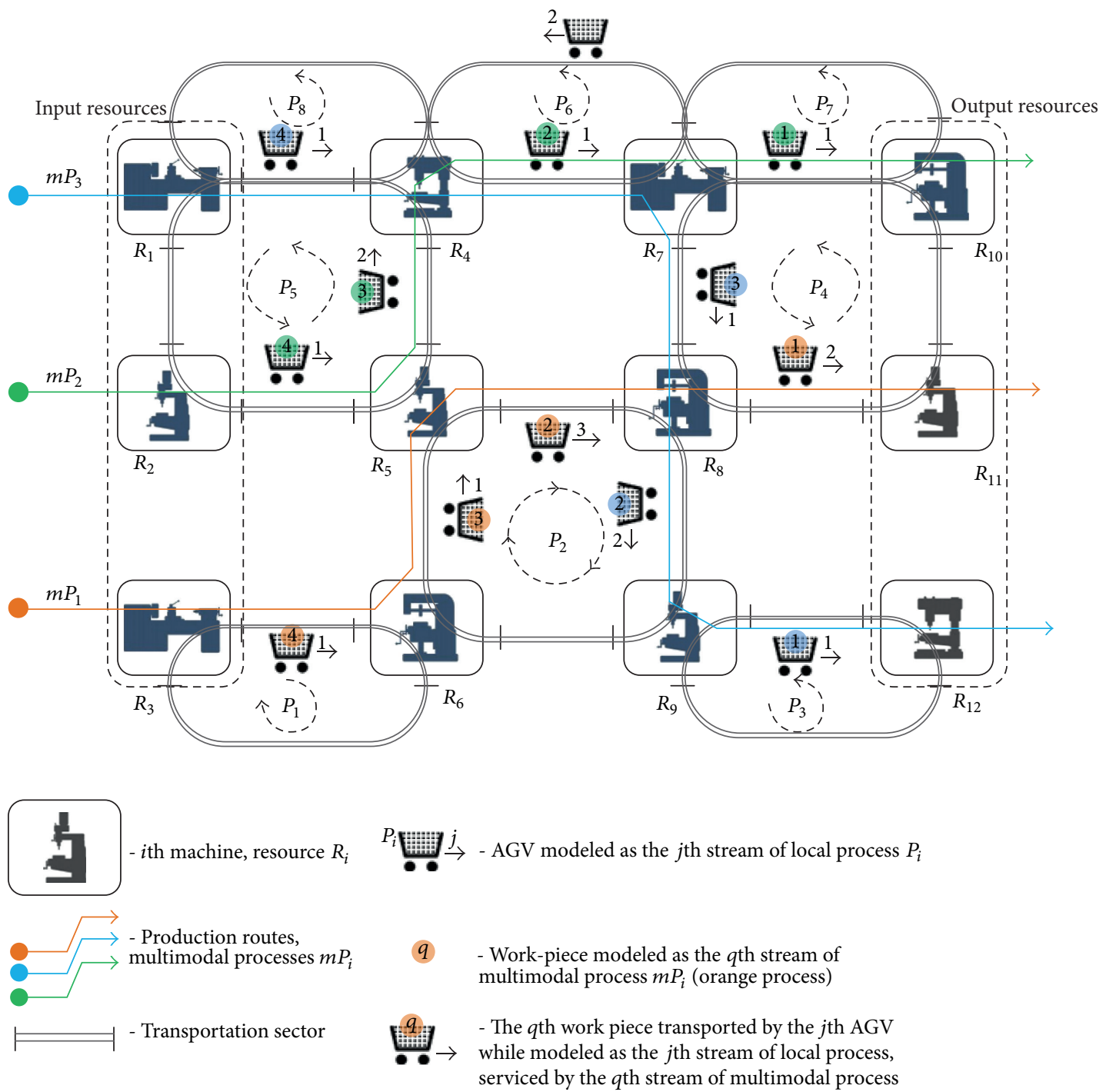

Figure 1: Example of an AGVS.

Similarly the cyclic multimodal processes $m P_{1}, m P_{2}$, and $m P_{3}$ follow the routes (see Figure 2) as follows:

$$
\begin{gathered}
m p_{1}^{k}=\left(R_{3}, R_{13}, R_{6}, R_{17}, R_{5}, R_{21}, R_{8}, R_{22}, R_{11}\right), \\
k=1, \ldots, 4, \\
m p_{2}^{k}=\left(R_{2}, R_{20}, R_{5}, R_{24}, R_{4}, R_{28}, R_{7}, R_{29}, R_{10}\right), \\
k=1, \ldots, 4, \\
m p_{3}^{k}=\left(R_{1}, R_{27}, R_{4}, R_{28}, R_{7}, R_{25}, R_{8}, R_{18}, R_{9}, R_{15}, R_{12}\right), \\
k=1, \ldots, 4 .
\end{gathered}
$$

Let us assume that $m p_{1}^{k}, m p_{2}^{k}$, and $m p_{3}^{k}$ can be seen as follows (see Figure 2):

$$
\begin{array}{r}
m p_{1}^{k}=\left(\left(R_{3}, R_{13}, R_{6}\right),\left(R_{17}, R_{5}\right),\left(R_{21}, R_{8}\right),\left(R_{22}, R_{11}\right)\right), \\
k=1, \ldots, 4,
\end{array}
$$

$$
\begin{aligned}
& m p_{2}^{k}=\left(\left(R_{2}, R_{20}, R_{5}\right),\left(R_{24}, R_{4}\right),\left(R_{28}, R_{7}\right),\left(R_{29}, R_{10}\right)\right), \\
& k=1, \ldots, 4, \\
& m p_{3}^{k}=\left(\left(R_{1}, R_{27}, R_{4}\right),\left(R_{28}, R_{7}\right),\left(R_{25}, R_{8}\right),\right. \\
&\left.\left(R_{18}, R_{9}\right),\left(R_{15}, R_{12}\right)\right), \quad k=1, \ldots, 4,
\end{aligned}
$$

where one has the following: $\left(R_{3}, R_{13}, R_{6}\right),\left(R_{17}, R_{5}\right),\left(R_{21}, R_{8}\right)$, and $\left(R_{22}, R_{11}\right)$-subsequences of routes $p_{1}^{1}, p_{2}^{1}, p_{2}^{3}$, and $p_{4}^{2}$ defining the transportation sections of $m p_{1}^{k} ;\left(R_{2}, R_{20}, R_{5}\right)$, $\left(R_{24}, R_{4}\right),\left(R_{28}, R_{7}\right)$, and $\left(R_{29}, R_{10}\right)$-subsequences of routes $p_{5}^{1}, p_{5}^{2}, p_{6}^{1}$, and $p_{7}^{1}$ defining the transportation sections of $m p_{2}^{k}$; and $\left(R_{1}, R_{27}, R_{4}\right),\left(R_{28}, R_{7}\right),\left(R_{25}, R_{8}\right),\left(R_{18}, R_{9}\right)$, and $\left(R_{15}, R_{12}\right)$-subsequences of routes $p_{8}^{1}, p_{6}^{2}, p_{4}^{1}, p_{2}^{2}$, and $p_{3}^{1}$ defining the transportation sections of $m p_{3}^{k}$.

Let us assume also that the considered SCCPs follow the constraints stated below [15]: 


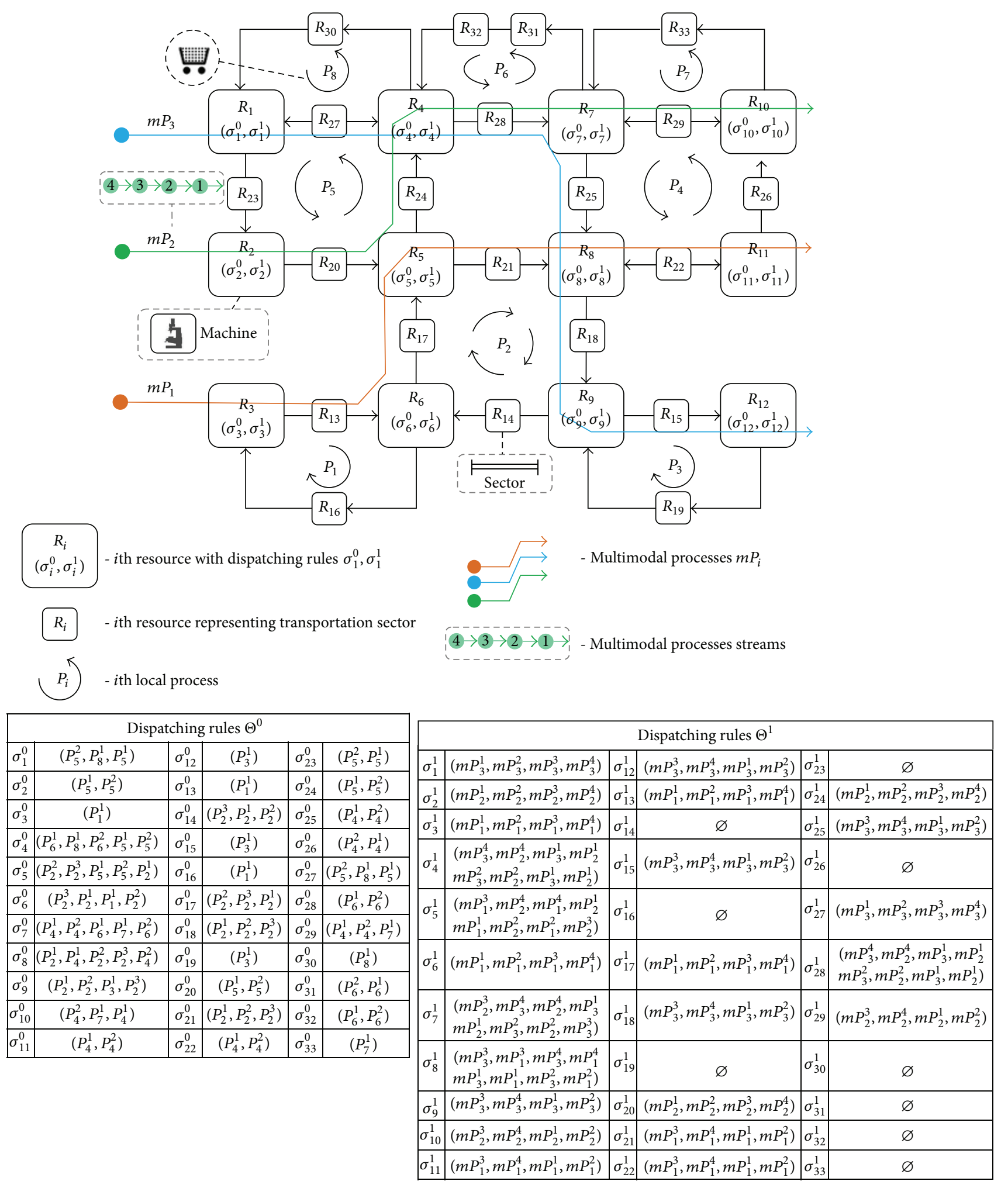

FIGURE 2: Example of FMS-SCCP model of an AGVS.

(i) a new subsequent operation of the local process may start on a required resource only if the current operation has been completed and the resource has been released; (ii) each new consecutive operation in the multimodal process may start its execution on an assigned resource only if the current operation has been completed and the resource has been released and an 
appropriate local process begins its consecutive operation on this resource;

(iii) local/multimodal processes share common resources in the mutual exclusion mode, the operation of a local/multimodal process can only be suspended if the necessary resource is occupied, suspended processes cannot be released, and processes are nonperceptible; that is, a resource may not be taken from a process as long as it is used by it;

(iv) the SCCP resources can be shared by local and multimodal processes as well as by both of them;

(v) multimodal processes encompassing production flow conveyed by AGVs follow local transportation routes;

(vi) different multimodal processes can be executed simultaneously along the same local process;

(vii) local and multimodal processes execute cyclically with periods $\alpha$ and $m \alpha$, respectively; resources occur uniquely in each transportation route;

(viii) in a cyclic steady state, each $i$ th stream must cover its local route the same number of times.

A resource conflict (caused by the application of the mutual exclusion protocol) is resolved with the aid of a priority dispatching rule [14-16], which determines the order in which streams access shared resources. For instance, in the case of the resource $R_{5}$, the priority dispatching rule $\sigma_{5}^{0}=$ $\left(P_{2}^{2}, P_{2}^{3}, P_{5}^{1}, P_{5}^{2}, P_{2}^{1}\right)$ determines the order in which streams of local processes can access the shared resource $R_{5}$; in the case considered the stream $P_{2}^{2}$ is allowed to access as first, then the stream $P_{2}^{3}$ as next, then streams $P_{5}^{1}, P_{5}^{2}$, and $P_{2}^{1}$, and then once again $P_{2}^{2}$, and so on. The stream $P_{i}^{k}$ occurs the same number of times (in the considered system once) in each dispatching rule associated with the resources featuring in its route (in the case considered each stream $P_{i}^{k}$ occurs uniquely). The SCCP shown in Figure 2 is specified by the following set of dispatching rules: $\Theta=\left\{\Theta^{0}, \Theta^{1}\right\}$, where $\Theta^{0}=\left\{\sigma_{1}^{0}, \ldots, \sigma_{33}^{0}\right\}$ is set of rules determining the orders of local processes and $\Theta^{1}=\left\{\sigma_{1}^{1}, \ldots, \sigma_{33}^{1}\right\}$ is set of rules determining the orders of multimodal processes.

In general, the following notation is used.

(i) A sequence $p_{i}^{k}=\left(p_{i, 1}^{k}, p_{i, 2}^{k}, \ldots, p_{i, j}^{k}, \ldots, p_{i, l r(i)}^{k}\right)$ specifies the route of the stream of a local process $P_{i}^{k}$ (the $k$ th stream of the $i$ th local process $P_{i}$ ). Its components define the resources used in the execution of operations, where $p_{i, j}^{k} \in R$ (the set of resources $\left.R=\left\{R_{1}, R_{2}, \ldots, R_{c}, \ldots, R_{m}\right\}\right)$ denotes the resources used by the $k$ th stream of the $i$ th local process in the $j$ th operation; in the rest of the paper, the $j$ th operation executed on the resource $p_{i, j}^{k}$ in the stream $P_{i}^{k}$ will be denoted by $o_{i, j}^{k} ; \operatorname{lr}(i)$ is the length of the cyclic process route (all streams of $P_{i}$ are of the same length). For example, the route $p_{1}^{1}=\left(R_{16}, R_{3}, R_{13}, R_{6}\right)$ of the stream of process $P_{1}$ (Figure 2 ) is the sequence $p_{1}^{1}=\left(p_{1,1}^{1}, p_{1,2}^{1}, p_{1,3}^{1}, p_{1,4}^{1}\right)$, where the first element $p_{1,1}^{1}$ is equal to $R_{16}$, whereas the second, $p_{1,2}^{1}=R_{3}$, and so on, $p_{1,3}^{1}=R_{13}, p_{1,4}^{1}=R_{6}$.

(ii) $x_{i, j}^{k}(l) \in \mathbb{N}$ is the timing of commencement of operation $o_{i, j}^{k}$ in the $l$ th cycle.

(iii) $t_{i}^{k}=\left(t_{i, 1}^{k}, t_{i, 2}^{k}, \ldots, t_{i, j}^{k}, \ldots, t_{i, l r(i)}^{k}\right)$ specifies the operation times of local processes, where $t_{i, j}^{k}$ denotes the time of execution of operation $o_{i, j}^{k}$.

(iv) $m p_{i}^{k}=\left(m p r_{i_{1}}^{q_{1}}\left(a_{i_{1}}, b_{i_{1}}\right), m p r_{i_{2}}^{q_{2}}\left(a_{i_{2}}, b_{i_{2}}\right), \ldots, m p r_{i_{y}}^{q_{y}}\left(a_{i_{y}}\right.\right.$, $\left.b_{i_{y}}\right)$ ) specifies the route of the stream $m P_{i}^{k}$ from the multimodal process $m P_{i}$ (the $k$ th stream of the $i$ th multimodal process $m P_{i}$ ), where

$m p r_{i}^{q}(a, b)$

$\begin{cases}\left(p_{i, a}^{q}, p_{i, a+1}^{q}, \ldots, p_{i, b}^{q}\right), & a \leq b, \\ \left(p_{i, a}^{q}, p_{i, a+1}^{q}, \ldots, p_{i, l r(i)}^{q}, p_{i, 1}^{q}, \ldots, p_{i, b-1}^{q}, p_{i, b}^{q}\right) & a>b, \\ a, b \in\{1, \ldots, \operatorname{lr}(i)\},\end{cases}$

is the subsequence of the route $p_{i}^{q}=\left(p_{i, 1}^{q}, p_{i, 2}^{q}, \ldots\right.$, $\left.p_{i, j}^{q}, \ldots, p_{i, l r(i)}^{q}\right)$ containing elements from $p_{i, a}^{q}$ to $p_{i, b}^{q}$.

In other words, the transportation route $m p_{i}$ is a sequence of parts of routes of local processes. For instance, the route followed by process $m P_{1}$ (see Figure 2) is as follows: $m p_{1}^{k}=\left(\left(R_{3}, R_{13}, R_{6}\right)\right.$, $\left.\left(R_{17}, R_{5}\right),\left(R_{21}, R_{8}\right),\left(R_{22}, R_{11}\right)\right)$, where $m p r_{1}^{1}(2,4)=$ $\left(R_{3}, R_{13}, R_{6}\right) ; \operatorname{mpr}_{2}^{1}(7,8)=\left(R_{17}, R_{5}\right) ; \operatorname{mpr}_{2}^{3}(1,2)=$ $\left(R_{21}, R_{8}\right), m p r_{4}^{2}(5,6)=\left(R_{22}, R_{11}\right)$.

(v) $m x_{i, j}^{k}(l) \in \mathbb{N}$ is the timing of commencement of operation $m o_{i, j}^{k}$ in the $l$ th cycle.

(vi) $m t_{i}^{k}=\left(m t_{i, 1}^{k}, m t_{i, 2}^{k}, \ldots, m t_{i, j}^{k}, \ldots, m t_{i, l m(i)}^{k}\right)$ specifies the operation times of multimodal processes, where $m t_{i, j}^{k}$ denotes the time of execution of operation $m o_{i, j}^{k}$ and $\operatorname{lm}(i)$ is the length of the cyclic process route $m p_{i}^{k}$.

(vii) $\Theta=\left\{\Theta^{0}, \Theta^{1}\right\}$ is the set of priority dispatching rules, $\Theta^{i}=\left\{\sigma_{1}^{i}, \sigma_{2}^{i}, \ldots, \sigma_{c}^{i}, \ldots, \sigma_{m}^{i}\right\}$ is the set of priority dispatching rules for local $(i=0) /$ multimodal $(i=1)$ processes where $\sigma_{c}^{i}=\left(s_{c, 1}^{i}, \ldots, s_{c, d}^{i}, \ldots, s_{c, l p(c)}^{i}\right)$ are sequence components which determine the order in which the processes can be executed on the resource $R_{c}, s_{c, d}^{0} \in H$ (where $H$ is the set of local streams, e.g., in the case presented in Figure $2, H=\left\{P_{1}^{1}, P_{2}^{1}\right.$, $\left.\left.P_{2}^{2}, P_{2}^{3}, P_{3}^{1}, P_{4}^{1}, P_{4}^{2}, P_{5}^{1}, P_{5}^{2}, P_{6}^{1}, P_{6}^{2}, P_{7}^{1}\right\}\right)$, and $s_{c, d}^{1} \in m H$ (where $m H$ is the set of multimodal streams, e.g., in case from Figure 2, $m H=\left\{m P_{1}^{k}, m P_{2}^{k}, m P_{3}^{k} \mid k=\right.$ $1 \cdots 4\})$.

Using the above notation, a SCCP can be defined as a tuple:

$$
\mathrm{SC}=((R, \mathrm{SL}), \mathrm{SM}),
$$


where one has the following: $R=\left\{R_{1}, R_{2}, \ldots, R_{c}, \ldots, R_{m}\right\}-$ the set of resources, $m$-the number of resources, $\mathrm{SL}=\left(U, T, \Theta^{0}\right)$-the structure of local processes, that is, $U=\left\{p_{1}^{1}, \ldots, p_{1}^{l_{s(1)}}, \ldots, p_{n}^{1}, \ldots, p_{n}^{l s(n)}\right\}$-the set of routes of local process, $l s(i)$ - the number of streams belonging to the process $P_{i}, n$-the number of local processes, $T=\left\{t_{1}^{1}, \ldots, t_{1}^{l s(1)}, \ldots, t_{n}^{1}, \ldots, t_{n}^{l_{s(n)}}\right\}$ - the set of sequences of operation times in local processes, $\Theta^{0}=\left\{\sigma_{1}^{0}, \sigma_{2}^{0}, \ldots, \sigma_{c}^{0}\right.$, $\left.\ldots, \sigma_{m}^{0}\right\}$-the set of priority dispatching rules for local processes, $\mathrm{SM}=\left(M, m T, \Theta^{1}\right)$-the structure of multimodal processes, that is, $M=\left\{m p_{1}^{1}, \ldots, m p_{1}^{l s m(1)}, \ldots, m p_{w}^{1}, \ldots\right.$, $\left.m p_{w}^{l s m(n)}\right\}$-the set of routes of a multimodal process, $\operatorname{lsm}(i)$-the number of streams belonging to the process $m P_{i} w$-the number of multimodal processes, $m T=\left\{m t_{1}^{1}, \ldots, m t_{w}^{l s m(1)}, \ldots, m t_{n}^{1}, \ldots, m t_{w}^{l s m(n)}\right\}$ - the set of sequences of operation times in multimodal processes, and $\Theta^{1}=\left\{\sigma_{1}^{1}, \sigma_{2}^{1}, \ldots, \sigma_{c}^{1}, \ldots, \sigma_{m}^{1}\right\}$ - the set of priority dispatching rules for multimodal processes.

The SCCP model (5) can be seen as a multilevel model, cf. Figure 3, that is, a model composed of an " $R$ level" (resources), an "SL level” (local cyclic processes), and an "SM" level" (multimodal cyclic processes), as well as an "SM${ }^{q}$ level" (the qth metamultimodal process). The SL level is defined by the transportation routes structure following the set $U$ of local processes and the set of parameters $\Theta$ determining the required system behavior. In turn, the $\mathrm{SM}^{1}$ level takes into account multimodal processes, as well as metamultimodal processes $\left(\mathrm{SM}^{2}\right.$ level) composed of multimodal processes from the $\mathrm{SM}^{1}$ level. In other words, it is assumed that the variables describing $\mathrm{SM}^{q}$ are the same as in the case of $\mathrm{SM}$, whereas the routes of the multimodal process of the $q$ th level remain composed of the processes from the $(q-1)$ th level. The presented model is an extended version of a simplified model limited to $R$ and SL levels, which is introduced in [15].

Therefore, in general, the SC $=((R, \mathrm{SL}), \mathrm{SM})$ model can be seen as composed of $l p$ levels as follows:

$$
\mathrm{SC}^{l p}=\left(\left(\left(\left((R, \mathrm{SL}), \mathrm{SM}^{1}\right), \mathrm{SM}^{2}\right), \ldots, \mathrm{SM}^{q}\right), \ldots, \mathrm{SM}^{l p}\right)
$$

The SC ${ }^{l p}$ model emphasizes structural characteristics of the SCCP modeled. In turn, the behavioral characteristics can be specified in terms of the admissible states reachability concept, that is, either taking into account the state space concept including both cyclic steady states and leading to them transient states [16] or the cyclic steady state space only $[14,15]$.

The second way, which does not take into account initial states leading through the transient states to the cyclic steady states, seems to be quite close to the cyclic scheduling methods widely used in many real-life cases $[9,11]$.

In that context the relevant multilevel cyclic schedule $X^{l p}$ encompassing the SCCP behavior on each of its processes level, that is, local SL and multimodal $\mathrm{SM}^{l p}$ processes, can be defined as follows:

$$
\begin{gathered}
X^{l p}=\left(\left(\left(\left((X, \alpha),\left(m^{1} X, m^{1} \alpha\right)\right), \ldots,\right), \ldots,\left(m^{q} X, m^{q} \alpha\right)\right),\right. \\
\left.\ldots,\left(m^{l p} X, m^{l p} \alpha\right)\right),
\end{gathered}
$$

where one has the following: $X, \alpha /\left(m^{q} X, m^{q} \alpha\right)$-sequence of commencement of operations and periodicity of local/multimodal (ith level) processes executions.

It should also be noted that the schedule $X^{l p}$ can be defined as a sequence of ordered pairs describing behaviors of local $(X, \alpha)$ and multimodal $\left(m^{i} X, m^{i} \alpha\right)$ processes, where $X$ is a sequence of timings $x_{i, j}^{k}$ (for $l=0$ th cycle) of commencement of operations $o_{i, j}^{k}$ from streams $P_{i}^{k}$ executed along local processes $X=\left(x_{1,1}^{1}, x_{1,2}^{1}, \ldots, x_{n, l r(n)}^{l s(n)}\right)$, and by analogy, the sequence $m^{q} X=\left(m^{q} x_{1,1}^{1}, m^{q} x_{1,2}^{1}, \ldots, m^{q} x_{w(q), \operatorname{lm}(w, q)}^{\operatorname{lsm}(w, q)}\right)$ consists of the timing of commencement of multimodal processes operations (from the qth level, where one has the following: $w(q)$-the number of multimodal processes at the $q$ th level, $\operatorname{lsm}(i, q)$ - the number of streams of the $i$ th process at the $q$ th level, and $\operatorname{lm}(i, q)$ - the number of operation of the $i$ th process at the $q$ th level).

Variables $x_{i, j}^{k} / m^{q} x_{i, j}^{k} \in \mathbb{Z}$ describe the timing of commencement of operations in the $q$ th cycle of the SCCP cyclic steady state behavior: $x_{i, j}^{k}(l)=x_{i, j}^{k}+l \cdot \alpha / m^{q} x_{i, j}^{k}(l)=m^{q} x_{i, j}^{k}+$ $l \cdot m^{q} \alpha$.

Since values of $x_{i, j}^{k} / m^{q} x_{i, j}^{k}$ follow from system structure, hence the cyclic behavior $X^{l p}$ of SCCP is determined by its structure $\mathrm{SM}^{l p}$. Moreover, the multimodal processes behavior $\left(m^{i} X, m^{i} \alpha\right)$ also depends on the local cyclic processes behavior $(X, \alpha)$.

In the general case, besides depending on the structural characteristics of SCCP the values of the considered variables $x_{i, j}^{k} / m^{q} x_{i, j}^{k}$ depend on constraints from the mutual exclusion protocol, that is, the set of priority dispatching rules $\Theta$, operation times, and so forth $[14,15]$ as well as on the way the local and multimodal processes interacting with each other. For example, in case of the two levels structure model, that is, including levels SL and SM as shown in Figure 2, the constraints determining $x_{i, j}^{k} / m^{q} x_{i, j}^{k}$ can be expressed by the following rules [15]:

(i) for local processes: the timing of commencement of operation $o_{i, j}^{k}$ beginning states for a maximum of the completion time of operation $o_{i, j-1}^{k}$ preceding $o_{i, j}^{k}$ and the release time (with delay $\Delta t$ ) of the resource $p_{i, j}^{k}$ awaiting for $o_{i, j}$ execution is as follows:

moment of operation $o_{i, j}^{k}$ beginning

$$
\begin{aligned}
=\max \left\{\left(\text { moment of } p_{i, j}^{k} \text { release }+ \text { lag time } \Delta t\right),\right. \\
\left.\quad\left(\text { moment of operation } o_{i, j-1}^{k} \text { completion }\right)\right\} \\
i=1, \ldots, n ; \quad j=1, \ldots, \operatorname{lr}(i) ; \quad k=1, \ldots, l s(i) ;
\end{aligned}
$$




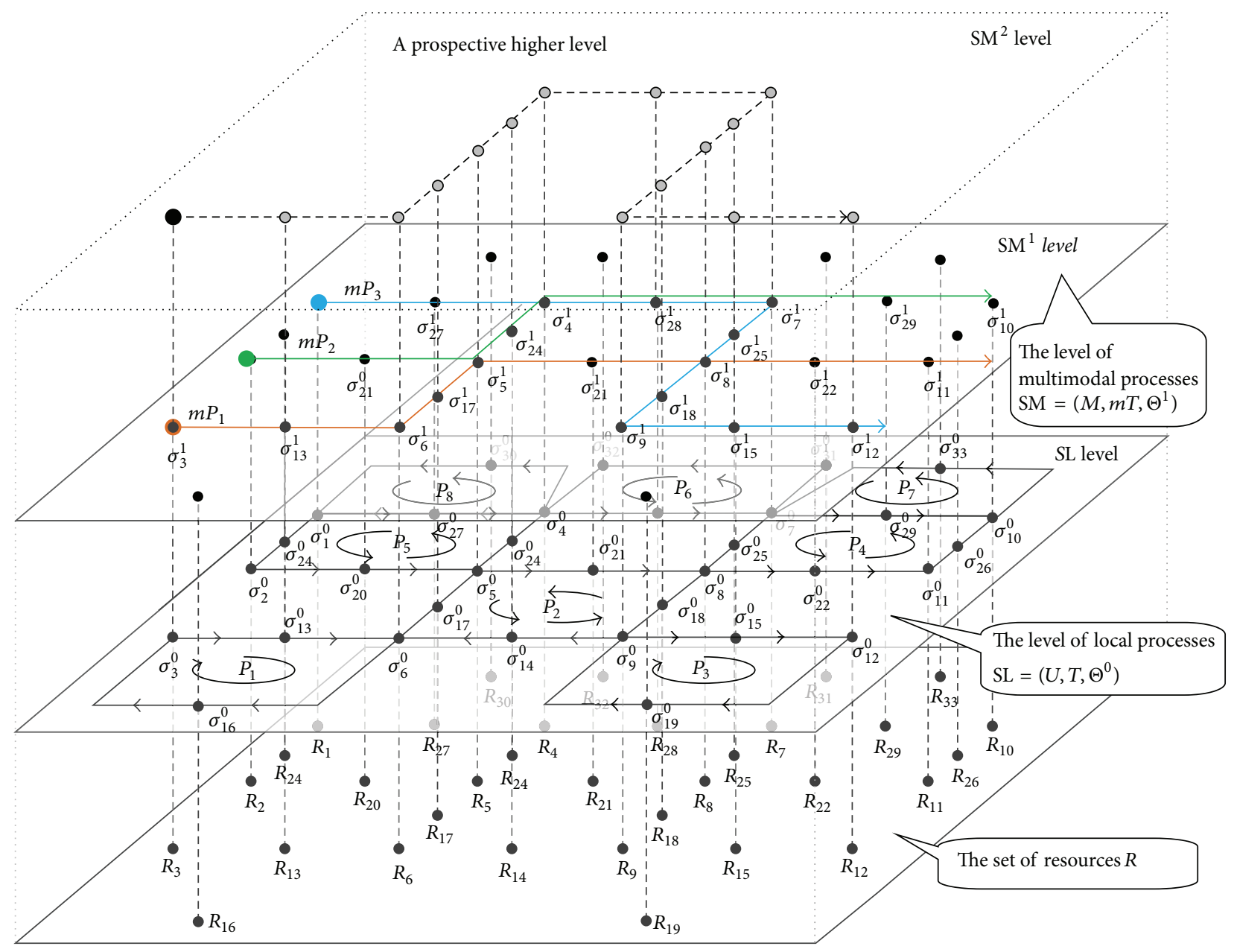

FIGURE 3: Multilayered model of the SCCP from Figure 2.

(ii) for multimodal processes: the timing of commencement of operation $m o_{i, j}^{k}$ beginning is equal to the nearest admissible value (determined by set $\mathscr{X}_{i, j}^{k}$ of values $m x_{i, j}^{k}$ ) being a maximum of both the completion time of operation $m o_{i, j-1}^{k}$ preceding $m o_{i, j}$ and the release time (lag time $\Delta t m$ ) of the resource $m p_{i, j}^{k}$ awaiting for $m o_{i, j}$ execution. Consider

moment of operation $m o_{i, j}^{k}$ beginning $=\left\lceil\max \left\{\left(\right.\right.\right.$ moment of $m p_{i, j}^{k}$ release + lag time $\left.\Delta t m\right)$, moment of operation $m o_{i, j-1}^{k}$ completion $\}\left.\right|_{X_{i, j}^{k}}$ $i=1, \ldots, n ; \quad j=1, \ldots, \operatorname{lm}(i) ; \quad k=1, \ldots, \operatorname{lsm}(i)$

where one has the following: $\mathscr{X}_{i, j}^{k}$-set of values $m x_{i, j}^{k}$ following the set of local processes $X,\left(X_{i, j}^{k}\right.$-the set of moments when operation $m o_{i, j}^{k}$ of multimodal process may use required local process) and $\lceil a\rceil_{B}-$ the smallest integer greater than or equal to $a$ in terms of the set $B:\lceil a\rceil_{B}=\min \{k \in B: k \geq a\}$.

In other words, the timing of commencement of operation $m o_{i, j}$ belongs to the set $\mathscr{X}_{i, j}^{k}$ and being coincident with operation processes by relevant local transportation process $P_{i}$.

The constraints determining (due to introduced rules (8) and (9)) the timing of commencement of operations for the SCCP from Figure 2 are gathered in Tables 1 and 2.

2.2. Cyclic Steady State Space. According to the previous section, a set of cyclic process achieved in the structure $\mathrm{SC}^{l p}$ (6) can be represented as a cyclic schedule $X^{l p}(7)$ that meets the constraints (8) and (9). Figure 4 presents an example of a cyclic schedule which can be achieved in the system illustrated in Figure 1. It shows that for a set of production plan (represented by multimodal processes $m P_{1}, m P_{2}$, and $\mathrm{mP}_{3}$ ) it is possible to organize the work of AGVs responsible for transportation of elements between workstations (local processes $P_{1}-P_{8}$ ) in such a way that no deadlocks appear in the system or there is no process waiting at the resources. An 
TABLE 1: Constraints determining the local processes behavior of the SCCP from Figure 2.

\begin{tabular}{|c|c|c|}
\hline \multicolumn{3}{|c|}{ Constraints of the local processes } \\
\hline $\begin{array}{l}x_{1,1}^{1}=x_{1,4}^{1}+t_{1,4}^{1}-\alpha \\
x_{1,2}^{1}=x_{1,1}^{1}+t_{1,1}^{1} \\
x_{1,3}^{1}=x_{1,2}^{1}+t_{1,2}^{1} \\
x_{1,4}^{1}=\max \left\{\left(x_{2,7}^{1}+\Delta t\right),\left(x_{1,3}^{1}+t_{1,3}^{1}\right)\right\} \\
- \\
x_{2,1}^{1}=\max \left\{\left(x_{2,6}^{3}+\Delta t-\alpha\right),\left(x_{2,8}^{1}+t_{2,8}^{1}-\alpha\right)\right\} \\
x_{2,2}^{1}=\max \left\{\left(x_{4,7}^{1}+\Delta t-\alpha\right),\left(x_{2,1}^{1}+t_{2,1}^{1}\right)\right\} \\
x_{2,3}^{1}=\max \left\{\left(x_{2,8}^{3}+\Delta t-\alpha\right),\left(x_{2,2}^{1}+t_{2,2}^{1}\right)\right\} \\
x_{2,4}^{1}=\max \left\{\left(x_{2,1}^{3}+\Delta t\right),\left(x_{2,3}^{1}+t_{2,3}^{1}\right)\right\} \\
x_{2,5}^{1}=\max \left\{\left(x_{2,3}^{3}+\Delta t\right),\left(x_{2,4}^{1}+t_{2,4}^{1}\right)\right\} \\
x_{2,6}^{1}=\max \left\{\left(x_{2,3}^{3}+\Delta t\right),\left(x_{2,5}^{1}+t_{2,5}^{1}\right)\right\} \\
x_{2,7}^{1}=\max \left\{\left(x_{2,4}^{3}+\Delta t\right),\left(x_{2,6}^{1}+t_{2,6}^{1}\right)\right\} \\
x_{2,8}^{1}=\max \left\{\left(x_{5,7}^{2}+\Delta t\right),\left(x_{2,7}^{1}+t_{2,7}^{1}\right)\right\} \\
- \\
x_{2,1}^{2}=\max \left\{\left(x_{2,8}^{1}+\Delta t-\alpha\right),\left(x_{2,8}^{2}+t_{2,8}^{2}-\alpha\right)\right\} \\
x_{2,2}^{2}=\max \left\{\left(x_{2,1}^{1}+\Delta t\right),\left(x_{2,1}^{2}+t_{2,1}^{2}\right)\right\} \\
x_{2,3}^{2}=\max \left\{\left(x_{2,2}^{2}+\Delta t\right),\left(x_{2,2}^{2}+t_{2,2}^{2}\right)\right\} \\
x_{2,4}^{2}=\max \left\{\left(x_{4,5}^{1}+\Delta t\right),\left(x_{2,3}^{2}+t_{2,3}^{2}\right)\right\} \\
x_{2,5}^{2}=\max \left\{\left(x_{2,4}^{1}+\Delta t\right),\left(x_{2,4}^{2}+t_{2,4}^{2}\right)\right\} \\
x_{2,6}^{2}=\max \left\{\left(x_{2,5}^{1}+\Delta t\right),\left(x_{2,5}^{2}+t_{2,5}^{2}\right)\right\} \\
x_{2,7}^{2}=\max \left\{\left(x_{2,1}^{1}+\Delta t\right),\left(x_{2,6}^{2}+t_{2,6}^{2}\right)\right\} \\
x_{2,8}^{2}=\max \left\{\left(x_{1,1}^{1}+\Delta t-\alpha\right),\left(x_{2,7}^{2}+t_{2,7}^{2}\right)\right\} \\
- \\
x_{2,1}^{3}=\max \left\{\left(x_{2,8}^{2}+\Delta t-\alpha\right),\left(x_{2,8}^{3}+t_{2,8}^{3}-\alpha\right)\right\} \\
x_{2,2}^{3}=\max \left\{\left(x_{2,1}^{2}+\Delta t\right),\left(x_{2,1}^{3}+t_{2,1}^{3}\right)\right\} \\
x_{2,3}^{3}=\max \left\{\left(x_{2,2}^{2}+\Delta t\right),\left(x_{2,2}^{3}+t_{2,2}^{3}\right)\right\} \\
x_{2,4}^{3}=\max \left\{\left(x_{2,3}^{2}+\Delta t\right),\left(x_{2,3}^{3}+t_{2,3}^{3}\right)\right\} \\
x_{2,5}^{3}=\max \left\{\left(x_{2,4}^{2}+\Delta t\right),\left(x_{2,4}^{3}+t_{2,4}^{3}\right)\right\} \\
x_{2,6}^{3}=\max \left\{\left(x_{2,5}^{2}+\Delta t\right),\left(x_{2,5}^{3}+t_{2,5}^{3}\right)\right\} \\
x_{2,7}^{3}=\max \left\{\left(x_{2,6}^{3}+\Delta t\right),\left(x_{2,6}^{3}+t_{2,6}^{3}\right)\right\} \\
x_{2,8}^{3}=\max \left\{\left(x_{2,7}^{2}+\Delta t\right),\left(x_{2,7}^{3}+t_{2,7}^{3}\right)\right\}\end{array}$ & $\begin{array}{l}x_{4,1}^{2}=\max \left\{\left(x_{4,8}^{1}+\Delta t-\alpha\right),\left(x_{4,8}^{2}+t_{4,8}^{2}-\alpha\right)\right\} \\
x_{4,2}^{2}=\max \left\{\left(x_{4,1}^{1}+\Delta t\right),\left(x_{4,1}^{2}+t_{4,1}^{2}\right)\right\} \\
x_{4,3}^{2}=\max \left\{\left(x_{4,2}^{1}+\Delta t\right),\left(x_{4,2}^{2}+t_{4,2}^{2}\right)\right\} \\
x_{4,4}^{2}=\max \left\{\left(x_{4,3}^{1}+\Delta t\right),\left(x_{4,3}^{2}+t_{4,3}^{2}\right)\right\} \\
x_{4,5}^{2}=\max \left\{\left(x_{4,4}^{1}+\Delta t\right),\left(x_{4,4}^{2}+t_{4,4}^{2}\right)\right\} \\
x_{4,6}^{2}=\max \left\{\left(x_{2,7}^{3}+\Delta t\right),\left(x_{4,5}^{2}+t_{4,5}^{2}\right)\right\} \\
x_{4,7}^{2}=\max \left\{\left(x_{4,6}^{1}+\Delta t\right),\left(x_{4,6}^{2}+t_{4,6}^{2}\right)\right\} \\
x_{4,8}^{2}=\max \left\{\left(x_{4,7}^{1}+\Delta t\right),\left(x_{4,7}^{2}+t_{4,7}^{2}\right)\right\} \\
- \\
x_{5,1}^{1}=\max \left\{\left(x_{5,5}^{2}+\Delta t-\alpha\right),\left(x_{5,8}^{1}+t_{5,8}^{1}-\alpha\right)\right\} \\
x_{5,2}^{1}=\max \left\{\left(x_{5,6}^{2}+\Delta t-\alpha\right),\left(x_{5,1}^{1}+t_{5,1}^{1}\right)\right\} \\
x_{5,3}^{1}=\max \left\{\left(x_{2,5}^{3}+\Delta t\right),\left(x_{5,2}^{1}+t_{5,2}^{1}\right)\right\} \\
x_{5,4}^{1}=\max \left\{\left(x_{5,8}^{2}+\Delta t-\alpha\right),\left(x_{5,3}^{1}+t_{5,3}^{1}\right)\right\} \\
x_{5,5}^{1}=\max \left\{\left(x_{6,4}^{1}+\Delta t\right),\left(x_{5,4}^{1}+t_{5,4}^{1}\right)\right\} \\
x_{5,6}^{1}=\max \left\{\left(x_{8,3}^{1}+\Delta t\right),\left(x_{5,5}^{1}+t_{5,5}^{1}\right)\right\} \\
x_{5,7}^{1}=\max \left\{\left(x_{8,2}^{1}+\Delta t\right),\left(x_{5,6}^{1}+t_{5,6}^{1}\right)\right\} \\
x_{5,8}^{1}=\max \left\{\left(x_{5,4}^{2}+\Delta t\right),\left(x_{5,7}^{1}+t_{5,7}^{1}\right)\right\} \\
- \\
x_{3,1}^{1}=x_{3,4}^{1}+t_{3,4}^{1}-\alpha \\
x_{3,2}^{1}=x_{3,1}^{1}+t_{3,1}^{1} \\
x_{3,3}^{1}=\max \left\{\left(x_{2,7}^{2}+\Delta t\right),\left(x_{3,2}^{1}+t_{3,2}^{1}\right)\right\} \\
x_{3,4}^{1}=x_{3,3}^{1}+t_{3,3}^{1} \\
- \\
x_{4,1}^{1}=\max \left\{\left(x_{7,4}^{1}+\Delta t-\alpha\right),\left(x_{4,8}^{1}+t_{4,8}^{1}-\alpha\right)\right\} \\
x_{4,2}^{1}=\max \left\{\left(x_{6,1}^{2}+\Delta t\right),\left(x_{4,1}^{1}+t_{4,1}^{1}\right)\right\} \\
x_{4,3}^{1}=\max \left\{\left(x_{4,6}^{2}+\Delta t-\alpha\right),\left(x_{4,2}^{1}+t_{4,2}^{1}\right)\right\} \\
x_{4,4}^{1}=\max \left\{\left(x_{2,3}^{1}+\Delta t\right),\left(x_{4,3}^{1}+t_{4,3}^{1}\right)\right\} \\
x_{4,5}^{1}=\max \left\{\left(x_{4,8}^{2}+\Delta t-\alpha\right),\left(x_{4,4}^{1}+t_{4,4}^{1}\right)\right\} \\
x_{4,6}^{1}=\max \left\{\left(x_{4,1}^{2}+\Delta t\right),\left(x_{4,5}^{1}+t_{4,5}^{1}\right)\right\} \\
x_{4,7}^{1}=\max \left\{\left(x_{4,2}^{1}+\Delta t\right),\left(x_{4,6}^{1}+t_{4,6}^{1}\right)\right\} \\
x_{4,8}^{1}=\max \left\{\left(x_{7,1}^{1}+\Delta t-\alpha\right),\left(x_{4,7}^{1}+t_{4,7}^{1}\right)\right\}\end{array}$ & $\begin{array}{l}- \\
x_{5,1}^{2}=\max \left\{\left(x_{5,7}^{1}+\Delta t-\alpha\right),\left(x_{5,8}^{2}+t_{5,8}^{2}-\alpha\right)\right\} \\
x_{5,2}^{2}=\max \left\{\left(x_{5,8}^{1}+\Delta t-\alpha\right),\left(x_{5,1}^{2}+t_{5,1}^{2}\right)\right\} \\
x_{5,3}^{2}=\max \left\{\left(x_{5,1}^{1}+\Delta t\right),\left(x_{5,2}^{2}+t_{5,2}^{2}\right)\right\} \\
x_{5,4}^{2}=\max \left\{\left(x_{5,2}^{1}+\Delta t\right),\left(x_{5,3}^{2}+t_{5,3}^{2}\right)\right\} \\
x_{5,5}^{2}=\max \left\{\left(x_{5,3}^{1}+\Delta t\right),\left(x_{5,4}^{2}+t_{5,4}^{2}\right)\right\} \\
x_{5,6}^{2}=\max \left\{\left(x_{5,4}^{1}+\Delta t\right),\left(x_{5,5}^{2}+t_{5,5}^{2}\right)\right\} \\
x_{5,7}^{2}=\max \left\{\left(x_{5,5}^{1}+\Delta t\right),\left(x_{5,6}^{2}+t_{5,6}^{2}\right)\right\} \\
x_{5,8}^{2}=\max \left\{\left(x_{5,6}^{1}+\Delta t\right),\left(x_{5,7}^{2}+t_{5,7}^{2}\right)\right\} \\
- \\
x_{6,1}^{1}=\max \left\{\left(x_{6,3}^{2}+\Delta t-\alpha\right),\left(x_{6,5}^{1}+t_{6,5}^{1}-\alpha\right)\right\} \\
x_{6,2}^{1}=\max \left\{\left(x_{5,1}^{2}+\Delta t\right),\left(x_{6,1}^{1}+t_{6,1}^{1}\right)\right\} \\
x_{6,3}^{1}=\max \left\{\left(x_{6,5}^{2}+\Delta t-\alpha\right),\left(x_{6,2}^{1}+t_{6,2}^{1}\right)\right\} \\
x_{6,4}^{1}=\max \left\{\left(x_{4,5}^{2}+\Delta t\right),\left(x_{6,3}^{1}+t_{6,3}^{1}\right)\right\} \\
x_{6,5}^{1}=\max \left\{\left(x_{6,2}^{2}+\Delta t\right),\left(x_{6,4}^{1}+t_{6,4}^{1}\right)\right\} \\
- \\
x_{6,1}^{2}=\max \left\{\left(x_{6,3}^{2}+\Delta t\right),\left(x_{6,5}^{2}+t_{6,5}^{2}-\alpha\right)\right\} \\
x_{6,2}^{2}=\max \left\{\left(x_{6,2}^{1}+\Delta t\right),\left(x_{6,1}^{2}+t_{6,1}^{2}\right)\right\} \\
x_{6,3}^{2}=\max \left\{\left(x_{8,4}^{1}+\Delta t\right),\left(x_{6,2}^{2}+t_{6,2}^{2}\right)\right\} \\
x_{6,4}^{2}=\max \left\{\left(x_{6,4}^{1}+\Delta t\right),\left(x_{6,3}^{2}+t_{6,3}^{2}\right)\right\} \\
x_{6,5}^{2}=\max \left\{\left(x_{7,3}^{1}+\Delta t\right),\left(x_{6,4}^{2}+t_{6,4}^{2}\right)\right\} \\
- \\
x_{7,1}^{1}=x_{7,4}^{1}+t_{7,4}^{1}-\alpha \\
x_{7,2}^{1}=\max \left\{\left(x_{6,5}^{1}+\Delta t\right),\left(x_{7,1}^{1}+t_{7,1}^{1}\right)\right\} \\
x_{7,3}^{1}=\max \left\{\left(x_{4,4}^{2}+\Delta t\right),\left(x_{7,2}^{1}+t_{7,2}^{1}\right)\right\} \\
x_{7,4}^{1}=\max \left\{\left(x_{4,3}^{2}+\Delta t\right),\left(x_{7,3}^{1}+t_{7,3}^{1}\right)\right\} \\
- \\
x_{8,1}^{1}=\max \left\{\left(x_{5,3}^{2}+\Delta t\right),\left(x_{8,4}^{1}+t_{8,4}^{1}-\alpha\right)\right\} \\
x_{8,2}^{1}=\max \left\{\left(x_{5,2}^{2}+\Delta t\right),\left(x_{8,1}^{1}+t_{8,1}^{1}\right)\right\} \\
x_{8,3}^{1}=\max \left\{\left(x_{6,3}^{1}+\Delta t\right),\left(x_{8,2}^{1}+t_{8,2}^{1}\right)\right\} \\
x_{8,4}^{1}=x_{8,3}^{1}+t_{8,3}^{1}\end{array}$ \\
\hline
\end{tabular}

assumption was made that the transportation of work-pieces (streams of multimodal processes) takes one unit of time and their loading to/unloading from AGVs (local processes) takes place in the first and the last unit of each operation (see Figure 4).

The work [20] shows that schedules of this kind (ensuring such an organization of AGVs that guarantees the accomplishment of the set of production routes without any stoppages) can be obtained by means of solving the following constraint satisfaction problem (10):

$$
\mathrm{CS}_{X T}=\left(\left(\left\{X^{l p}, T^{l p}\right\},\left\{D_{X}, D_{T}\right\}\right),\left\{C_{L}, C_{M}\right\}\right),
$$

where one has the following: $X^{l p}, T^{l p}$-decision variables, $X^{l p}$-cyclic schedule (7), $T^{l p}$-sequence of operation times $T^{l p}=\left(\left(\left(T, m T^{1}\right), \ldots, m T^{q}\right), \ldots, m T^{l p}\right), D_{X}, D_{T}, D_{\alpha}=\mathbb{N}-$ domains determining admissible value of decision variables
$D_{X}: m^{q} x_{i, j}^{k}, x_{i, j}^{k} \in \mathbb{Z}, m^{q} \alpha, \alpha \in \mathbb{N} ; D_{T}: m^{q} t_{i, j}^{k}, t_{i, j}^{k} \in \mathbb{N}$, $\left\{C_{L}, C_{M}\right\}$ - the set of constraints $C_{L}$ and $C_{M}$ describing SCCP behavior, $C_{L}$-constraints determining cyclic steady state of local processes, that is, their cyclic schedule, and $C_{M}-$ constraints determining multimodal processes behavior.

The sequence of operations times $T^{l p}$ and the sequence of their beginning moments $X^{l p}$ both of them follow constraints $C_{L}, C_{M}$ which are the sufficient conditions for the SCCP cyclic steady state behavior, that is, the state following the problem (10) solution. In case of two level systems, that is, SL and SM (see Figure 3), the constraints $C_{L}, C_{M}$ can be specified in terms of constraints (8) and their extension (9) [15].

Presenting cyclic processes as $X^{l p}$ schedules is commonly used both in SCCP $[11,12,14,15]$ and in various problems of cyclic scheduling. Instead of cyclic schedules, the so-called behavior digraphs [16], that create the state space $\mathscr{P}$, can also be applied. 


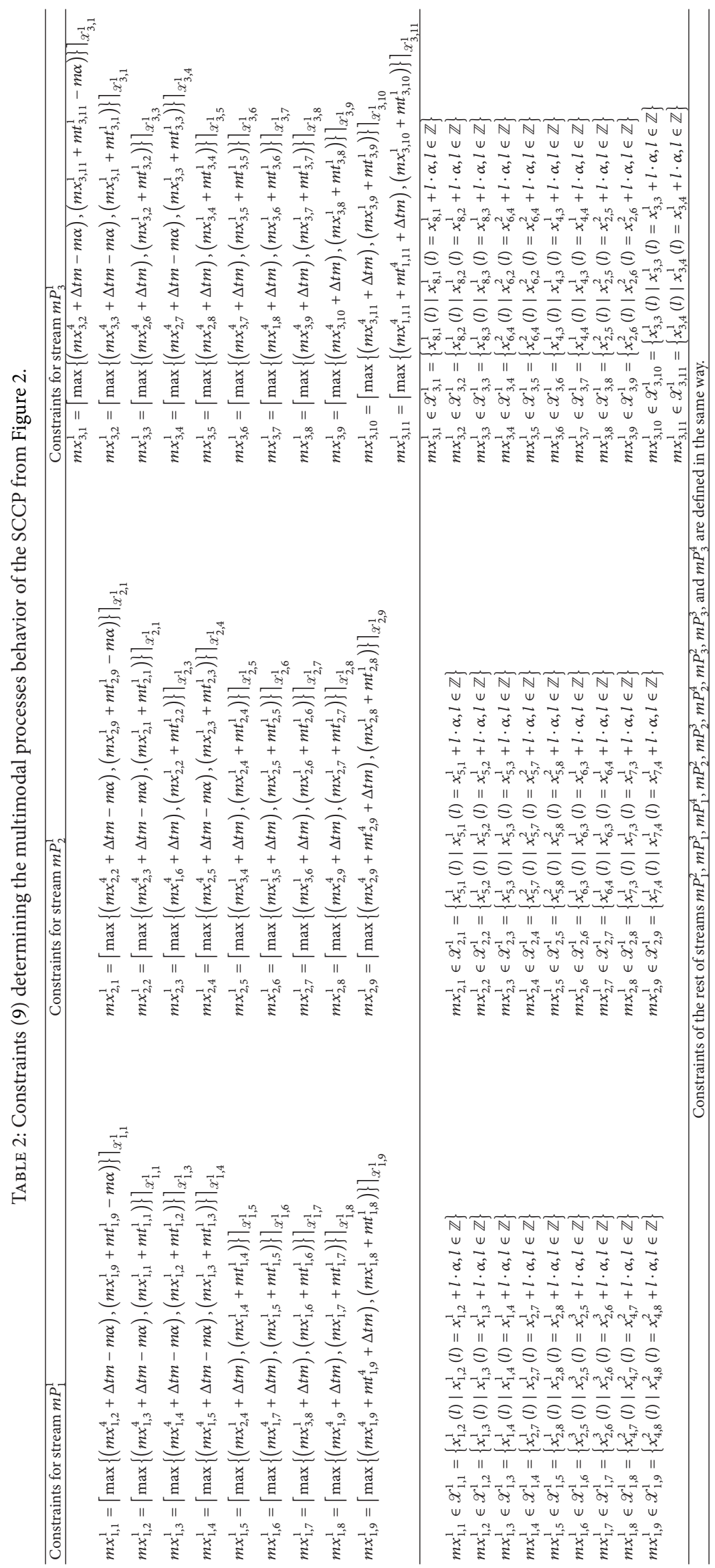



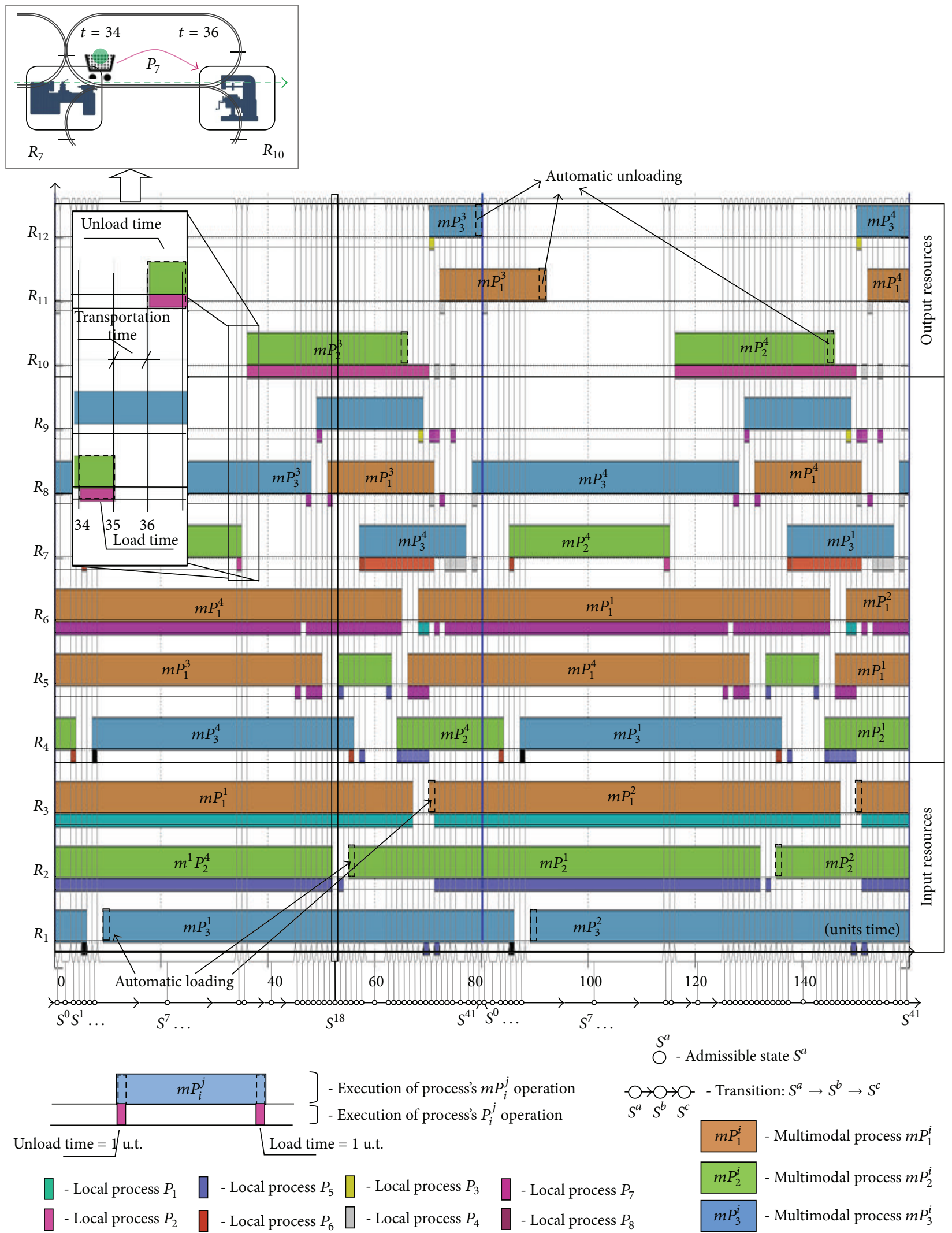

$\bigcirc \rightarrow \bigcirc-$ - Transition: $S^{a} \rightarrow S^{b} \rightarrow S^{c}$ $S^{a} S^{b} S^{c}$

\begin{tabular}{|c|c|}
\hline$m P_{1}^{i}$ & - Multimodal process $m P_{1}^{i}$ \\
\hline$m P_{2}^{i}$ & - Multimodal process $m P_{2}^{i}$ \\
\hline$m P_{3}^{i}$ & - Multimodal process $m l$ \\
\hline
\end{tabular}

FIGURE 4: AGVs fleet cyclic schedule matching the multiproduct manufacturing. 
The digraph $G_{W}$ (denoted by $W$ index, in case of cyclic processes representation) is a digraph whose vertexes represent the admissible states $S^{r}$ of the system and arcs represent transitions between the states (while depicting a given transition function $\delta[16]$ ).

In the considered case, the state of SCCP describes the present (valid in a given $t$ period) allocation on the resources of local (AGVs) and multimodal (technological routes) processes and describes the current access rights to the resources (determined by dispatching priority rules $\Theta$ ). Formally, in the state definition, three elements are distinguished that characterize processes at the particular behavior levels (see Figure 3). form:

In this approach, the state of SCCP takes the following

$$
S^{r}=\left(\left(\left(\left(S l^{r}, m^{1} S^{r}\right), \ldots, m^{l} S^{r}\right), \ldots\right), m^{l p} S^{r}\right),
$$

where one has the following: (i) $l p$-the number of levels of structure $\mathrm{SC}^{l p}(6)$ and (ii) $S l^{r}$ - the $r$ th state of local processes executed on the SL level (5) as follows:

$$
S l^{r}=\left(A^{r}, Z^{r}, Q^{r}\right),
$$

where one has the following: $A^{r}=\left(a_{1}{ }^{r}, a_{2}{ }^{r}, \ldots, a_{c}{ }^{r}, \ldots\right.$, $a_{m}{ }^{r}$ )-allocation of processes in the $r$ th state, $a_{c}{ }^{r} \in P \cup\{\Delta\}$, $a_{c}^{r}=P_{i}^{k}$-the $c$ th resource $R_{c}$ is occupied by the local stream $P_{i}^{k}$, and $a_{c}^{r}=\Delta$ - the $c$ th resource $R_{c}$ is unoccupied.

$Z^{r}=\left(z_{1}{ }^{r}, z_{2}{ }^{r}, \ldots, z_{c}{ }^{r}, \ldots z_{m}{ }^{r}\right)$ is the sequence of semaphores corresponding to the $r$ th state and $z_{c}{ }^{r} \in P$ is name of the stream (specified in the $c$ th dispatching rule $\sigma_{c}$, allocated to the $c$ th resource) which was allowed to occupy the $c$ th resource; for example, $z_{c}^{r}=P_{i}^{k}$ means that stream $P_{i}^{k}$ is currently allowed to occupy the $c$ th resource.

$Q^{r}=\left(q_{1}{ }^{r}, q_{2}{ }^{r}, \ldots, q_{c}{ }^{r}, \ldots, q_{m}{ }^{r}\right)$ is the sequence of semaphore indices, corresponding to the $r$ th state and $q_{c}{ }^{r}$ determines the position of the semaphore $z_{c}{ }^{r}$ in the priority dispatching rule $\sigma_{c}, z_{c}{ }^{r}=s_{c,\left(q_{c}{ }^{r}\right)}, q_{c}{ }^{r} \in \mathbb{N}$. For instance, $q_{2}{ }^{r}=2$ and $z_{2}{ }^{r}=P_{1}^{2}$ correspond to the semaphore $z_{2}{ }^{r}=P_{1}^{2}$ taking the 2 nd position in the priority dispatching rule $\sigma_{2}$.

(iii) $m^{l} S^{r}$ is the $r$ th state of multimodal processes executed on the $\mathrm{SM}^{l}$ level (6) as follows,

$$
m^{l} S^{r}=\left(m^{l} A^{r}, m^{l} Z^{r}, m^{l} Q^{r}\right)
$$

where one has the following: $m^{l} A^{r}=\left(m^{l} A_{1}^{r, 1}, \ldots\right.$, $\left.m^{l} A_{i}^{r, h}, \ldots, m^{l} A_{l w(l)}^{r, l s m(l w(l), l)}\right)$-allocation of the process streams $m^{l} P_{i}^{h}$; that is,

$$
m^{l} A_{i}^{r, h}=\left(m^{l} a_{i, 1}^{r, h}, m^{l} a_{i, 2}^{r, h}, \ldots, m^{l} a_{i, c}^{r, h}, \ldots, m^{l} a_{i, m}^{r, h}\right),
$$

where one has the following: $m^{l} P_{i}^{h}$-the $h$ th stream of the $i$ th multimodal process from $l$-level of $S^{l p}(6)$, $m$-the number of resources $R, m^{l} a_{i, c}^{r, h} \in\left\{m^{l} P_{i}^{h}, \Delta\right\}$, $m^{l} a_{i, c}^{r, h}=m^{l} P_{i}^{h}$ means that the $c$ th resource $R_{c}$ is occupied by the $h$ th stream of multimodal process $m^{l} P_{i}$, and $m^{l} a_{i, c}^{r, h}=\Delta$-the $c$ th resource $R_{c}$ which is released by the $h$ th stream of multimodal process $m^{l} P_{i}$.

$m^{l} Z^{r}=\left(m^{l} z_{1}^{r}, \ldots, m^{l} z_{c}^{r}, \ldots, m^{l} z_{m}{ }^{r}\right)$ and $m^{l} Q^{r}=\left(m^{l} q_{1}^{r}\right.$, $\left.\ldots, m^{l} q_{k}^{r}, \ldots, m^{l} q_{m}^{r}\right)$ are the sequences of semaphores and indices defined similarly as $Z^{r}$ and $Q^{r}$.

In the context of the introduced notions (allocation, semaphore, indexes) the $X^{l p}$ schedule presented in Figure 4 illustrates only the allocation of processes in time. The particular allocations are denoted by a frame and symbol " $\bigcirc$ " of the state $S^{r}$ connected with the given allocation. Therefore, the cyclic process represented by $X^{l p}$ schedule can be described with use of 42 states $\left(S^{0}-S^{41}\right)$.

The digraph $G_{W}=\left(V_{W}, E_{W}\right)$ corresponding to $X^{l p}$ schedule from Figure 4 was illustrated in Figure 5. The set of vertexes $V_{W}=\left\{S^{0}, \ldots, S^{41}\right\}$ includes all the states that can be achieved by the system in the course of implementing processes according to $X^{l p}$ schedule. The set of arcs $E_{W} \subseteq$ $V_{W} \times V_{W}$ defines the transitions between the states. Transition of the system from the state $S^{0}$ to the state $S^{1}$ (denoted by $S^{0} \rightarrow S^{1}$, and in Figure 5 as $\bigcirc \rightarrow \bigcirc$ ) occurs according to the transition function $\delta$, which was described in [16].

According to this approach, the digraph $G_{W}=\left(V_{W}, E_{W}\right)$ depicting the behavior from Figure 4 is a cycle including 42 vertexes; see Figure 5. Apart from $S^{r}$ states, Figure 5 illustrates also local states $\mathrm{Sl}^{r}$ (12) which only represent the behavior of local processes. In the discussed case, the number of local states is the same as the number of $S^{r}$ states. Generally, it does not have to be so, as there are situations when one local state is an element of numerous $S^{r}$ states $[14,15]$. The local states $S l^{r}$ as well as the digraph related to them should be perceived as a projection of $S^{r}$ states and $G_{W}$ digraph onto the level of local processes.

It should be noted that in the discussed example only one cyclic steady state is reachable, that is, as a result of the solution (10); that is, one schedule $X^{l p}$ was obtained along with one digraph $G_{W}$ corresponding to it. Generally, in the given structure $S^{l p}$ a lot of cyclic steady states can be reachable (which depends on the initial phases of dispatching rules) and therefore, we can consider the cyclic steady state space $\mathscr{P}$ as a set of digraphs that can be reachable in a given structure of $G_{W}$ digraphs.

The cyclic steady state space can be illustrated as a graph being the sum of all reachable digraphs: $\mathscr{P}=\left(V_{\mathscr{P}}, E_{\mathscr{P}}\right)=$ $G_{W, 1} \cup G_{W, 2} \cup \cdots \cup G_{W, l g}$ (where $G_{W, a} \cup G_{W, b}=\left(V_{a} \cup\right.$ $\left.V_{b}, E_{a} \cup E_{b}\right)$ ) are reachable in a given structure. There are also situations where in a given structure no cyclic steady states are reachable; then $\mathscr{P}=(\emptyset, \emptyset)$. The main reason for the lack of cyclic behaviors is the possible deadlocks.

The form of the space of states $\mathscr{P}$ is strictly dependent upon the form of $\mathrm{SC}^{l p}$ structure. In other words, the structure determines behaviors reachable in the system. Another example of a cyclic behavior is shown in 


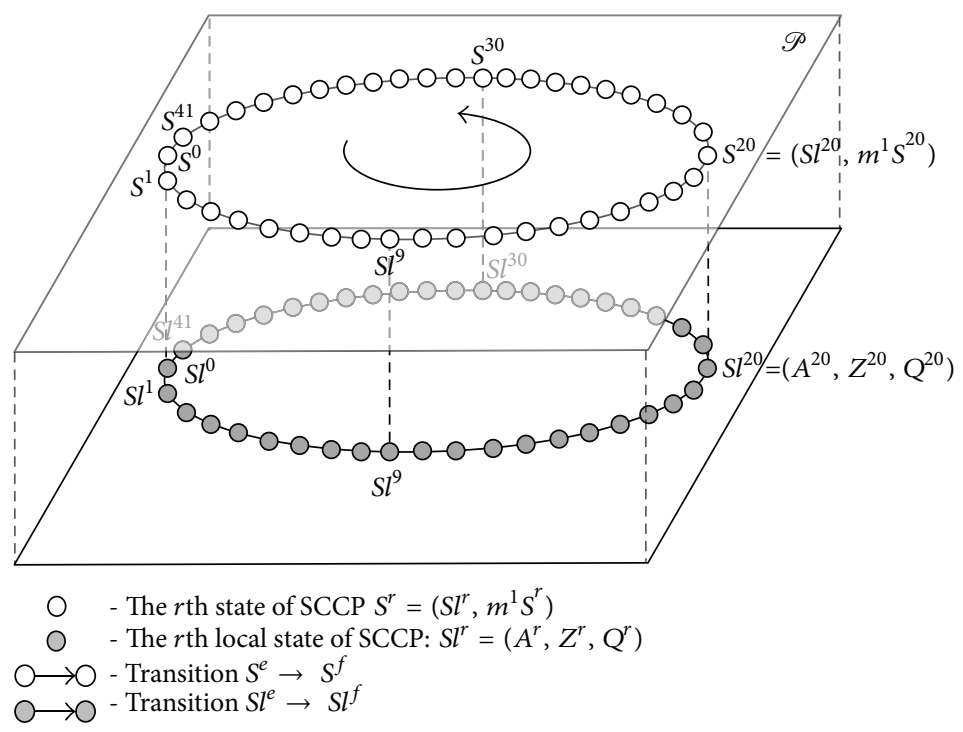

Figure 5: The digraph $G_{W}$ following Gantt's chart from Figure 4.

Figure 6. It is a cyclic schedule $X^{l p}$ reachable in SC structure (Figure 2) in which the manner of implementing production routes was changed (routes $m p_{2}^{k}=\left(\left(R_{2}\right.\right.$, $\left.\left.R_{20}, R_{5}\right),\left(R_{24}, R_{4}\right),\left(R_{28}, R_{7}\right),\left(R_{25}, R_{8}, R_{22}, R_{11}\right)\right)$ and $m p_{3}^{k}=$ $\left(\left(R_{1}, R_{27}, R_{4}\right),\left(R_{28}, R_{7}\right),\left(R_{29}, R_{10}\right)\right)$. There are still seven types of AGVs used for transport (local processes $P_{1}-P_{7}$ ) with routes remaining unchanged yet different than previously, when they performed their operations.

This situation can be interpreted as introducing a new production order into the system, which requires a new method of processing elements. The presence of cyclic schedule $X^{l p}$ (providing a solution to the problem (10)) and $G_{W}$ digraph (Figure 7 ) related to it mean that such an order can be implemented.

The presence of two different cyclic steady states leads to the question of their mutual reachability: Is it possible to smoothly change the system behavior from $G_{W, 1}$ to $G_{W, 2}$ (Figure 7)? In other words, is it possible to smoothly (with no need to stop the system) proceed from implementing the first production order $G_{W, 1}$ to the other $G_{W, 2}$ ?

\section{Multimodal Processes Rescheduling}

3.1. Problem Formulation. The question of the possibility of smoothly changing the cyclic behaviors of the system is related to the problem of mutual reachability of the two behavior digraphs $G_{W, 1}, G_{W, 2}$. The digraphs presented so far (Figure 7) are cycles. In general, a digraph may take the form of the so-called vortex $G_{S}$, that is, a digraph including a cycle representing a cyclic steady states $G_{W}$ and a tree $G_{T, i}$ representing transient states leading to cyclic steady states. An example of two vortexes $G_{S, 1}, G_{S, 2}$ is shown in Figure 8. Formally, the digraph of the vortex type is defined as follows:

$$
G_{S}=\left(V_{S}, E_{S}\right)=G_{W} \cup\left(\bigcup_{i=1}^{l t} G_{T, i}\right),
$$

where $\bigcup_{i=1}^{l t} G_{T, i}=G_{T, 1} \cup G_{T, 2} \cup \cdots \cup G_{T, l t}$, lt is the number of trees $G_{T, i}$ leading to $G_{W}$ cycle.

In this approach, the problem of mutual reachability of the behavior digraphs of mutual space of states is defined as follows: there is a given structure $\mathrm{SC}^{l p}$ and the cyclic steady state space $\mathscr{P}=\left(V_{\mathscr{P}}, E_{\mathscr{P}}\right)$ resulting from it, which includes two vortexes $G_{S, 1}, G_{S, 2}$ that represent two cyclic steady processes. It is therefore necessary to find answer to the following question: Are the digraphs $G_{W, 1}, G_{W, 2}$ (being subdigraphs of $G_{S, 1}, G_{S, 2}$ ) mutually reachable (Figure 8)? In the case they are mutually reachable the next question is how to make a transition between the digraphs?

In other words, the question of mutual reachability of cyclic behaviors can be treated as the question of the possibility of direct or indirect transition between the digraphs $G_{W, 1}$, $G_{W, 2}$. An example of such transitions is shown in Figure 8. An indirect transition should be understood as changing $G_{W, 1}$ into $G_{W, 2}$, resulting from the transition of the state $S^{x^{\prime}}$ into transitory path (a path of digraph $G_{T, i}$ ) leading to $G_{W, 2}$. The direct transition means a transition from the state $S^{x}$ right into the cycle $G_{W, 1}$.

\subsection{Searching for States with Mutual Allocation}

3.2.1. Sufficient Conditions. The possibility of transitions between cyclic steady states depends on the state $S^{x}\left(S^{x \prime}\right)$, which is an element of two digraphs: $G_{S, 1}$ and $G_{S, 2}$. In other words, it is necessary that in case of direct transition the following transitions between states occur:

(i) for all $S^{k} \in V_{W, 1}\left(S^{k} \rightarrow \cdots \rightarrow S^{x} \rightarrow \cdots \rightarrow S^{k}\right)$ for the digraph $G_{W, 1}$,

(ii) for all $S^{l} \in V_{W, 2}\left(S^{l} \rightarrow \cdots \rightarrow S^{x} \rightarrow \cdots \rightarrow S^{l}\right)$ for the digraph $G_{W, 2}$.

In SCCP, transitions of this kind are not acceptable. It results from the property saying that each state has at least 


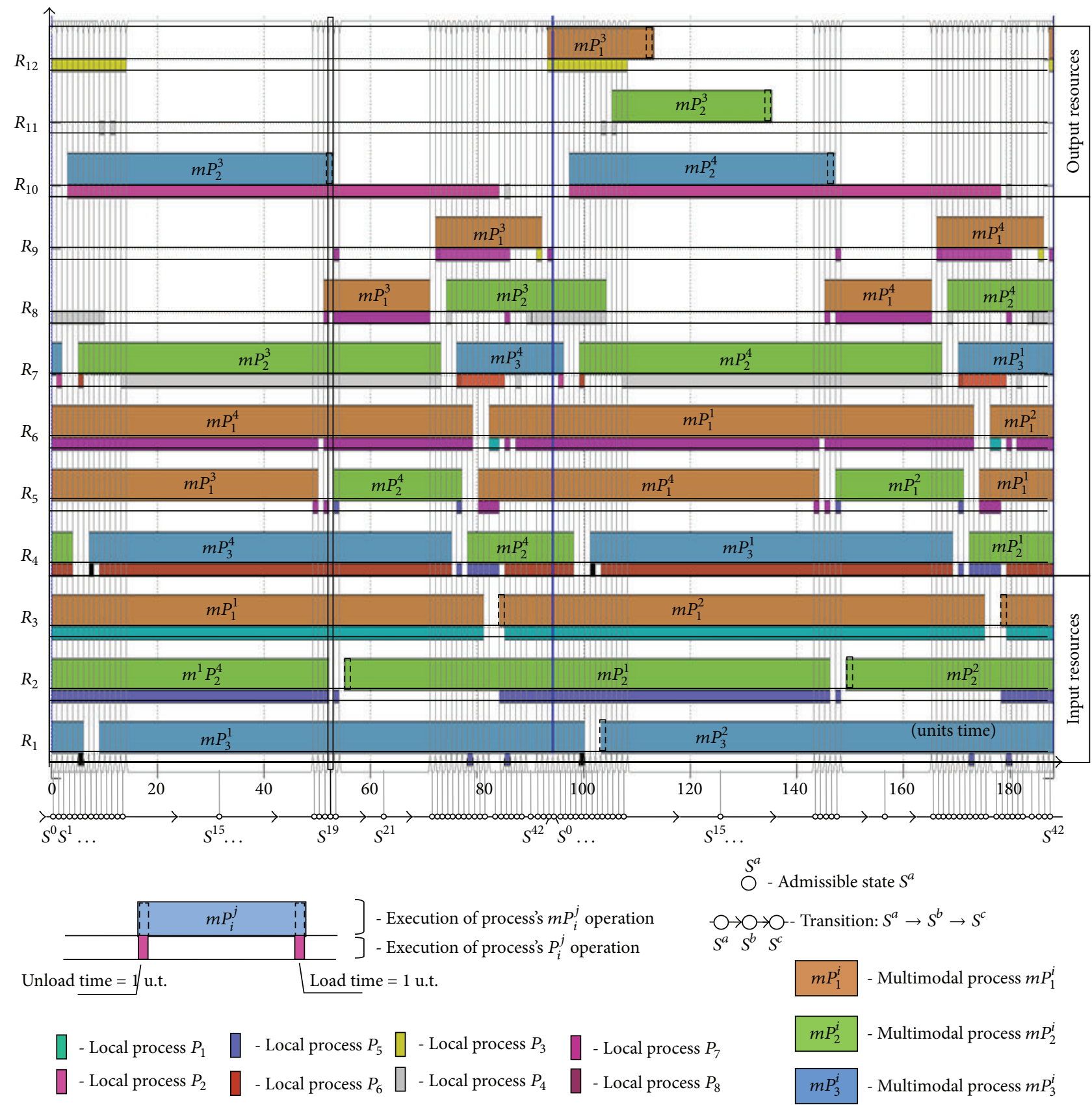

FIGURE 6: AGVs fleet cyclic schedule matching the multiproduct manufacturing for system with changed multimodal routes.

one descendant [16] (a descendant is the consecutive state after $S^{x}$ ). The presence of state $S^{x}$ included simultaneously into two digraphs $\left(G_{W, 1}, G_{W, 2}\right)$ would require the existence of at least two descendants of the state $S^{x}$.

Although there are no mutual states at the level of the space of states $\mathscr{P}$, the different projections of states upon lower levels of behavior as well as their components (space of allocation, semaphores, etc.) may be elements belonging to numerous digraphs at the same time.

An example of such a situation is shown in Figure 9, where the idea of a multilevel model of the cyclic steady states space $\mathscr{P}$ has been applied. The figure shows the projection of the space $\mathscr{P}$ upon the level of local processes behavior $\mathscr{P}_{\mathrm{SL}}$ (the second level in Figure 9) and upon the space of allocation $\mathscr{A}$ (the lowest level in Figure 9). The elements of the space $\mathscr{A}$ are the allocations $A^{r}$ of the local states $S l^{r}=\left(A^{r}, Z^{r}, Q^{r}\right)$ occurring in the space $\mathscr{P}_{\mathrm{SL}}=\left(V_{\mathrm{SL}}, E_{\mathrm{SL}}\right)$ (where $S l^{r} \in V_{\mathrm{SL}}$ ), which at the same time is the projection of the space of states $\mathscr{P}$.

It is worth mentioning that some allocations of the space $\mathscr{A}$ are simultaneously mutual for several states. For example, the allocation $A^{2} \in \mathscr{A}$ is at the same time an element of the state $S l^{3}=\left(A^{2}, Z^{3}, Q^{3}\right)$ and the state $S l^{4}=\left(A^{2}, Z^{4}, Q^{4}\right)$. In practice, it means that in both states the local processes 

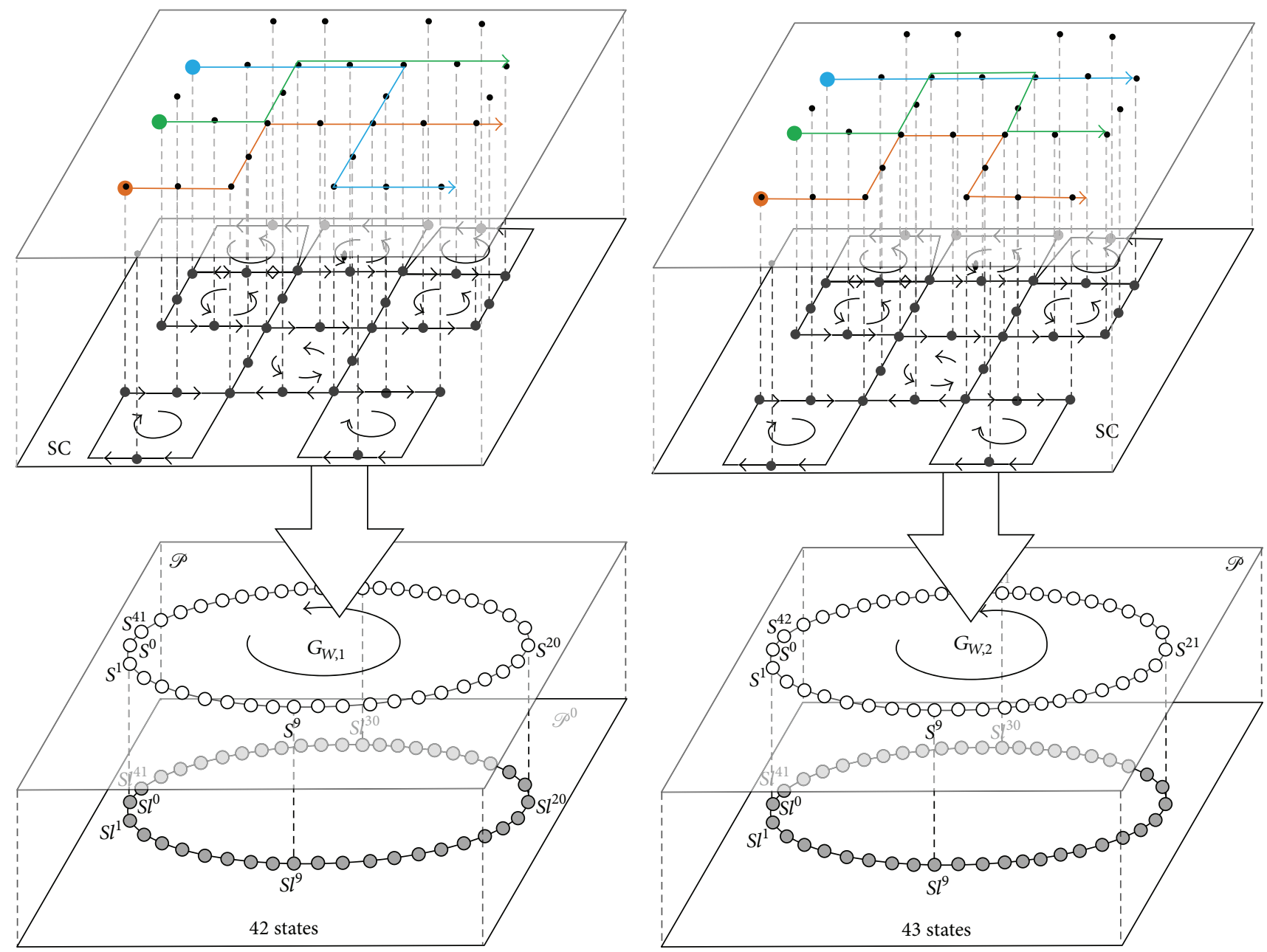

(a)

(b)

FIGURE 7: The state space for structures with different multimodal processes routes.

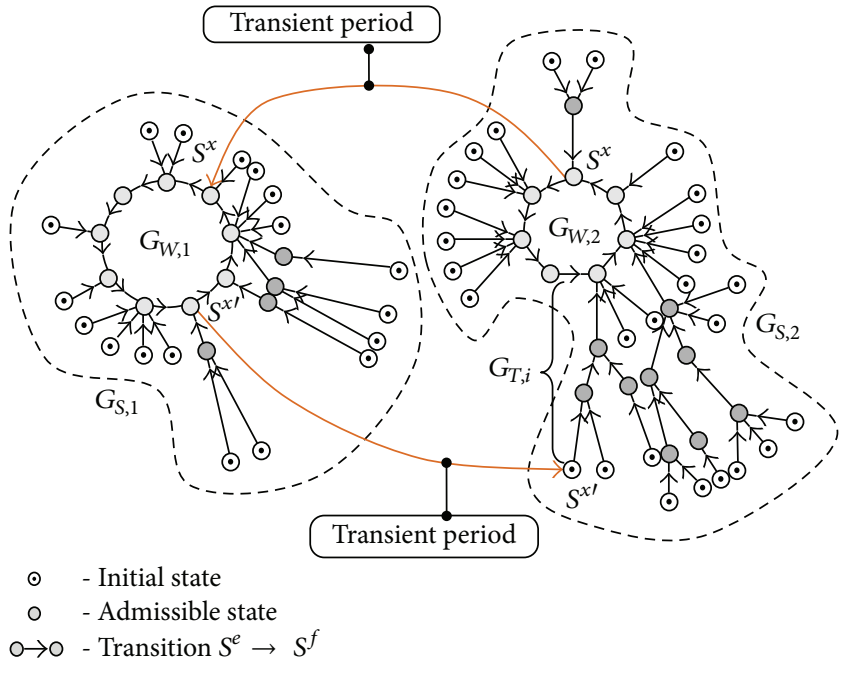

FIGURE 8: Illustration of the cyclic steady state space composed of two vortexes. (representing, e.g., means of transport such as AGVs) are allocated on the same resources. However, since there are various access rights (determined by $Z^{3}, Q^{3}$ and $Z^{4}, Q^{4}$ ) their further implantation will be different.

The existence of states of common allocation can be used for the transitions between various behavior digraphs. The states $S l^{3}$ and $S l^{4}$ differ only with the sequence of semaphores and indexes. Thus, if in the state $S l^{3}$ with allocation $A^{2}$, the form of $Z^{3}, Q^{3}$ changes into the form of $Z^{4}, Q^{4}$, we will attain the state $S l^{4}$ leading to another cycle.

The modification of the state $\mathrm{Sl}^{3}$ into $S l^{4}$ by changing the form of semaphores and indexes allows for an indirect transition between cyclic processes of the space $\mathscr{P}^{0}$. The direct transition is possible as a result of modifying the semaphores and indexes of the states $S l^{1}=\left(A^{1}, Z^{1}, Q^{1}\right)$ and $S l^{2}=\left(A^{1}, Z^{2}, Q^{2}\right)$ mutually sharing the allocation $A^{1}$. Therefore, the presence of states of mutual allocation implies the possibility of changing the set of cyclic steady states. 

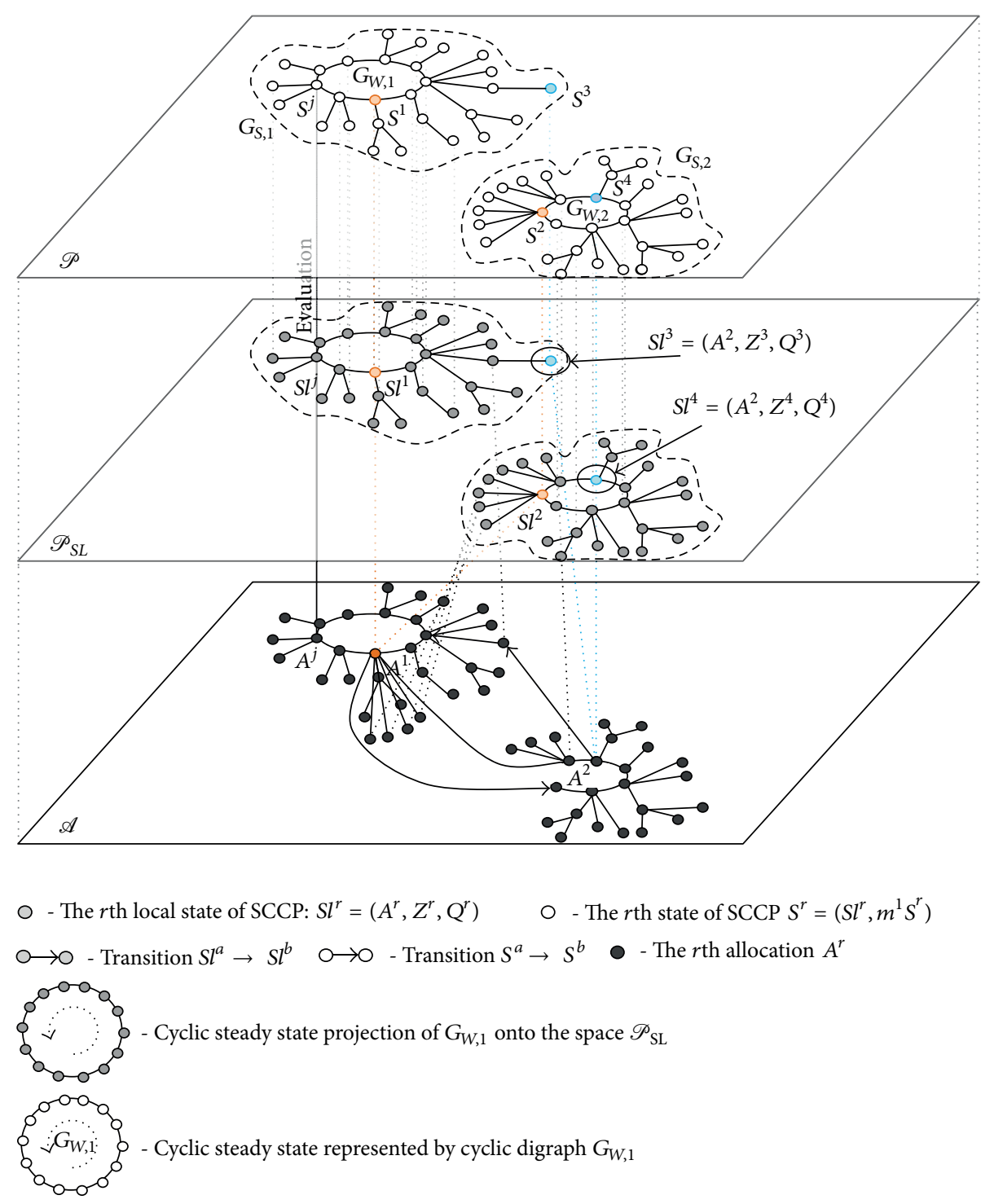

FIGURE 9: Illustration of cyclic steady state space projections, $\mathscr{P}_{\mathrm{SL}}$ and $\mathscr{A}$, for behavior digraphs from Figure 8.

The considered transitions between $\mathrm{Sl}^{3}$ and $S l^{4}$ as well as $S l^{1}$ and $S l^{2}$ refer mainly to digraphs occurring only at the local level (i.e., space $\mathscr{P}_{\mathrm{SL}}$ ). In case of digraphs $G_{S, 1}, G_{S, 2}$ of the space $\mathscr{P}$ a similar procedure should be followed. These states are the projections of the corresponding states from the space $\mathscr{P}$. The state $S l^{1}$ is the projection of the state $S^{1}=\left(S l^{1}, m^{1} S^{1}\right)$, which is an element of the digraph $G_{W, 1}$, and the state $S^{2}=$ $\left(S l^{2}, m^{1} S^{2}\right)$ is the projection of the digraph $G_{W, 2}$.

If, among states projecting upon $S l^{1}$ and $S l^{2}$, there are states (e.g., states $S l^{1}$ and $S l^{2}$ ) with mutual allocation $m^{1} A^{x}$ of the multimodal processes, the transition between $G_{W, 1}$ and $G_{W, 2}$ (and therefore also between $G_{S, 1}$ and $G_{S, 2}$ ) is possible as a result of modifications of corresponding semaphores and indexes (from the form of $m^{1} Z^{1}, m^{1} Q^{1}$ into the form of $m^{1} Z^{2}$, $\left.m^{1} Q^{2}\right)$.
The states $S^{1}$ and $S^{2}$ are characterized by mutual allocation of both local and multimodal processes. By means of modifying semaphores and indexes in the state $S^{1}$, a direct transition from digraph $G_{W, 1}$ to digraph $G_{W, 2}$ is possible.

In practice, the change of semaphores and indexes related to them means simply the change of the control rules (change of signaling) of the system, the moment the processes are allocated properly (compatible with $S^{1}$ ). The solution of this kind neither stops the implementation of the process (work of AGVs, technological routes) nor creates the need for changing their allocation (shift). In case of the modification of the state $S^{6}$ the transition is immediate and noninvasive; it only requires the change of control.

To sum up the above considerations, we can say that the transition between the two digraphs $G_{W, 1}$ and $G_{W, 2}$ is possible if they include states characterized by mutual allocation at 
every behavior level. This observation leads to the following two properties.

Property 1. Digraph $G_{W, 2}$ is reachable from the digraph $G_{W, 1}$ (which is denoted by $G_{W, 1} \rightarrow G_{W, 2}$ ) if there are states $S^{a} \epsilon$ $V_{W, 1}$ and $S^{b} \in V_{S, 2}$ (where $V_{W, 1}$ is the set of states of the digraph $G_{W, 1}$ and $V_{S, 2}$ is the set of states of the digraph $G_{S, 2}$ ) sharing mutual allocation of local and multimodal processes: $A^{a}=$ $A^{b} ; m^{l} A^{a}=m^{l} A^{b}$, for $l=1 \cdots l p$.

Property 2. Two digraphs $G_{W, 1}$ and $G_{W, 2}$ are mutually reachable (which is denoted by $G_{W, 1} \leftrightarrow G_{W, 2}$ ) if $G_{W, 1} \rightarrow G_{W, 2}$ and $G_{W, 2} \rightarrow G_{W, 1}$.

In this approach, the problem of digraphs reachability calls for an answer to the following question: Are there two states $S^{a} \in V_{W, 1}$ and $S^{b} \in V_{S, 2}$, sharing the same allocations $A^{a}=A^{b} ; m^{l} A^{a}=m^{l} A^{b}$, for $l=1 \cdots l p$, among states included into $G_{S, 1}$ and $G_{S, 2}$ ? If such states exist, the transition between the states is possible as a result of modifications of semaphores and indexes. In this context the direct transition is defined as

$$
\begin{aligned}
\cdots & \longrightarrow S^{a_{1}} \longrightarrow S^{a_{2}} \longrightarrow \cdots \longrightarrow S^{a_{(x-1)}} \\
& \longrightarrow\left(S^{a_{x}} \rightsquigarrow S^{b_{x}}\right) \longrightarrow S^{b_{(x+1)}} \longrightarrow \cdots
\end{aligned}
$$

and the indirect transition as

$$
\begin{gathered}
\cdots \longrightarrow S^{a_{1}} \longrightarrow \cdots \longrightarrow\left(S^{a_{x}} \rightsquigarrow S^{d_{x}}\right) \longrightarrow \cdots \\
\longrightarrow S^{d_{l d}} \longrightarrow S^{b_{j}} \longrightarrow S^{b_{(j+1)}} \cdots,
\end{gathered}
$$

where one has the following: $S^{a_{1}}, \ldots, S^{a_{(x-1)}}, S^{a_{x}}, \ldots, S^{a_{l a}}-$ states of digraph $G_{W, 1}, S^{b_{1}}, \ldots, S^{b_{(x-1)}}, S^{b_{x}}, \ldots, S^{b_{l b}}$-states of $\operatorname{digraph} G_{W, 2}, S^{d_{1}}, \ldots, S^{d_{x}}, S^{b_{(x+1)}} \ldots, S^{d_{l b}}$-states included into the transitory digraph leading $G_{T}$ to $G_{W, 2}, S^{a_{1}} \rightarrow S^{a_{2}}-$ transition between states with SCCP assumption and transitory function $\delta$ [16], $S^{a_{x}}, S^{b_{x}}$-states with mutual allocation, and $S^{a_{x}} \rightsquigarrow S^{b_{x}}$-transition from the state $S^{a_{x}}$ to $S^{b_{x}}$ as a result of states modification, consisting of a sequence change of semaphores and indexes from $Z^{a_{x}}, Q^{a_{x}}$ to $Z^{b_{x}}, Q^{a_{x}}$.

3.2.2. Searching Algorithm. In the situation when digraphs $G_{W, 1}$ and $G_{W, 2}$ and transitory processes leading to them are known (in other words, vortexes $G_{S, 1}$ and $G_{S, 2}$ (15) are known), determining the states with mutual allocation is not a complex task. Due to a small number of states (in practice no more than a few hundred states per vortex), all that has to be done is to successively compare all potential variants. An algorithm corresponding to such an approach looks as Algorithm 1.

In Algorithm 1, one has the following: $G_{S, 1}=\left(V_{S, 1}, E_{S, 1}\right)$, $G_{S, 2}=\left(V_{S, 2}, E_{S, 2}\right)$-input data, vortexes (15), $S^{a}, S^{b}$-states (15) included in the digraph $G_{W, 1}, G_{S, 2}$, and AC-set of pairs $\left(S^{a}, S^{b}\right)$ of states with mutual allocation.

The result of Algorithm 1 is the set AC including pairs of states $\left(S^{a}, S^{b}\right)$ with mutual allocations. The states can be used to determine the direct and indirect transitions between digraphs $G_{W, 1}$ and $G_{W, 2}$. Therefore, the existence of a nonempty set AC means a positive answer to the posed question.

To make things simple, an assumption can be made that we take into consideration that direct transitions and the digraphs have the same number of states (denoted by $l d$ ); thus computational complexity of Algorithm 1 is expressed by the function $f(l d)=l d^{2}$.

Algorithm 1 makes it possible to evaluate the mutual reachability of only two digraphs $G_{W, 1}$ and $G_{W, 2} \in$ DC (set of all cyclic digraphs existing in the space $\mathscr{P}$ ). In case of evaluating the mutual reachability of all digraphs from the set DC, it is necessary to make a search of this kind for a pair of processes. The computational complexity in such case amounts to

$$
f(l d, d c)=\frac{1}{2}\left(d c^{2}-d c\right) \cdot l d^{2}, \quad \text { where } d c=|\mathrm{DC}|
$$

Owing to polynomial character of the function of computational complexity, the problem of evaluating the mutual reachability of behavior digraphs is not a difficult one. All the computational effort is focused on the stage of determining cyclic digraphs (set DC), that is, solving the problem (10). In order to do so, methods described in $[11,12,14-16]$ can be applied.

Among the available methods, however, there is a deficit of those which can help determine transient states leading to cyclic digraphs (i.e., digraphs $G_{T}$ ). Their usability is therefore limited to evaluating direct transitions (where no information on transient states (processes) is required).

In order to determine the transient states, the method of vortex generation can be used. Vortexes determined with use of this method include cyclic processes as well as all transient processes leading to them. The example below illustrates the use of this method for evaluating mutual reachability of behavior digraphs.

3.3. Numerical Example. Consider two AGVs supporting multiproduct manufacturing flows and their SCCP models shown in Figures 2 and 7(b). Let us assume that the two series manufacturing of three different products follow the routes distinguished by different colored (green, orange, and blue) bold lines.

Each workstation can process only unique work-piece from a unique product kind at a given instance. Successive work-pieces following the product route of a given product kind while taking into account local material handling routes are treated as subsequent streams of manufacturing processes (in a given kind of product manufacturing flow) and are modeled as streams of multimodal processes. For instance, see streams of $m P_{1}, m P_{2}$, and $m P_{3}$ from Figure 2 as follows:

$$
\begin{array}{r}
m p_{1}^{k}=\left(\left(R_{3}, R_{13}, R_{6}\right),\left(R_{17}, R_{5}\right),\left(R_{21}, R_{8}\right),\left(R_{22}, R_{11}\right)\right), \\
k=1, \ldots, 4, \\
m p_{2}^{k}=\left(\left(R_{2}, R_{20}, R_{5}\right),\left(R_{24}, R_{4}\right),\left(R_{28}, R_{7}\right),\left(R_{29}, R_{10}\right)\right), \\
k=1, \ldots, 4,
\end{array}
$$




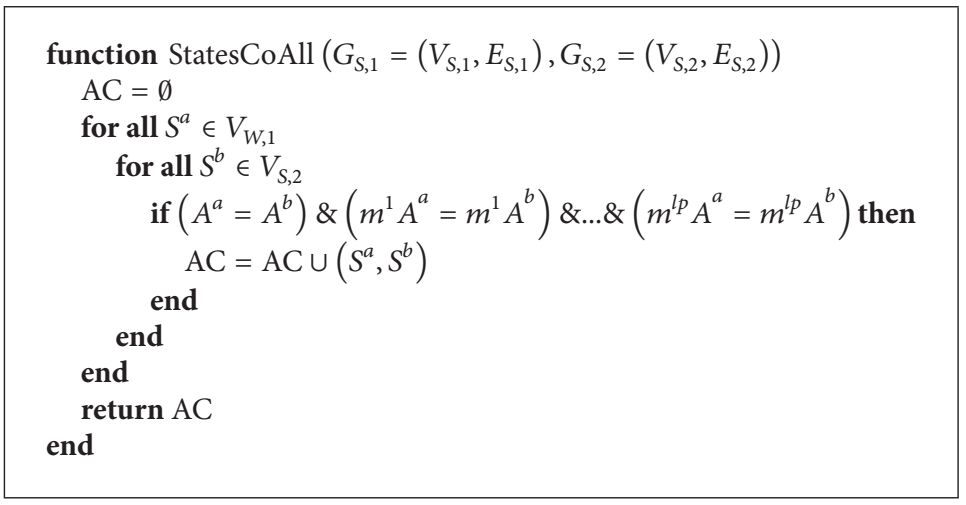

Algorithm 1

$$
\begin{aligned}
m p_{3}^{k}= & \left(\left(R_{1}, R_{27}, R_{4}\right),\left(R_{28}, R_{7}\right),\left(R_{25}, R_{8}\right),\left(R_{18}, R_{9}\right)\right. \\
& \left.\left(R_{15}, R_{12}\right)\right), \quad k=1, \ldots, 4
\end{aligned}
$$

and Figure 7(b) as follows:

$$
\begin{gathered}
m p_{1}^{k}=\left(\left(R_{3}, R_{13}, R_{6}\right),\left(R_{17}, R_{5}\right),\left(R_{21}, R_{8}\right),\left(R_{18}, R_{9}\right),\right. \\
\left.\left(R_{15}, R_{12}\right)\right), \quad k=1, \ldots, 4, \\
m p_{2}^{k}=\left(\left(R_{2}, R_{20}, R_{5}\right),\left(R_{24}, R_{4}\right),\left(R_{28}, R_{7}\right),\right. \\
\left.\left(R_{25}, R_{8}, R_{22}, R_{11}\right)\right), \quad k=1, \ldots, 3, \\
m p_{3}^{k}=\left(\left(R_{1}, R_{27}, R_{4}\right),\left(R_{28}, R_{7}\right),\left(R_{29}, R_{10}\right)\right), \quad k=1, \ldots, 3 .
\end{gathered}
$$

Both systems use the same fleet of 13 AGVs. A fleet supports work-pieces movements between workstations along the given manufacturing routes. The AGVs flows are modeled by streams $P_{1}^{1}, P_{2}^{1}, P_{2}^{2}, P_{2}^{3}, P_{3}^{1}, P_{4}^{1}, P_{4}^{2}, P_{5}^{1}, P_{5}^{2}, P_{6}^{1}, P_{6}^{2}, P_{7}^{1}$, and $P_{8}^{1}$ of local processes $P_{1}-P_{8}$. Each workstation (in general system resource) can be serviced only by one AGV at the moment. That means that both kinds of flow, that is, products and AGVs flows, are synchronized by a mutual exclusion protocol.

Given are the two cyclic schedules of multiproduct manufacturing supported by AVGs (see Figures 4 and 6) and corresponding to them two cyclic digraphs (Figures 7(a) and $7(b))$. Due to this schedules in first system four workpieces $m P_{i}^{1}, m P_{i}^{2}, m P_{i}^{3}, m P_{i}^{4}$ of each product $(i=1,2,3)$ are simultaneously processed in one cycle and one work-piece of each product is outputted at each 80 u.t. In second system four work-pieces of $m P_{1}^{k}$ and three work-pieces of $m P_{2}^{k}, m P_{3}^{k}$ product are simultaneously processed in one cycle and one work-piece of each product is outputted at each 96 u.t.

As mentioned before, operation times from the schedules considered take into account periods required for work-piece processing as well as material handling operations, that is, load/unload periods ( 1 u.t. for the work-piece load and 1 u.t. for its unload; see Figure 4). Moreover, the assumed time lags $\Delta t m$ (see (9) and constraints from Table 2) enable to take into account the work-pieces transportation between workstations. In the case considered all time lags are the same and equal to $\Delta t m=1$. Moreover, it is assumed that the input to $R_{1}$, $R_{2}$, and $R_{3}$ and the output of $R_{10}, R_{11}$, and $R_{12}$ workstations are loaded and unloaded by dedicated conveyors.

The operation times $m T$ and $T$ of considered systems are defined in Tables 3 and 4 . The timing $x_{i, j}^{k}, m x_{i, j}^{k}$ of commencement of operations following the constraints (8), (9) are presented in Tables 5 and 6.

The values presented in Tables 3-6 are the results of solving the problem (10), respectively, for the system from Figure 2 and Figure 7(b). In order to achieve this objective, OzMozart constraint programming environment was applied. The problem solving time in both cases did not exceed 3 seconds (Intel Core 2 Duo 3 GHz, RAM 4 GB).

During the analysis of digraphs of the attained schedules, it turned out that it is possible to smoothly change the behavior (with use of the state $S^{18}$ ) from the one presented in Figure 4 into the behavior from Figure 7. The transition was illustrated in Figures 11 and 12. In practice, a transition of this kind means the possibility of changing the current production order into a new one with no need to stop the system.

Therefore, a question may be posed whether an inverse situation is possible, when from the behavior represented by digraph $G_{W, 2}$, behavior $G_{W, 1}$ is attained (return to $G_{W, 1}$ ). In other words, the question is the following: Are the considered behavior digraphs mutually reachable, $G_{W, 1} \leftrightarrow G_{W, 2}$ ?

According to Property 2 it is possible if $G_{W, 1} \rightarrow G_{W, 2}$ and $G_{W, 2} \rightarrow G_{W, 1}$. The schedule shown in Figure 10 illustrates the reachability $G_{W, 1} \rightarrow G_{W, 2}$. In order to determine whether $G_{W, 2} \rightarrow G_{W, 1}$ is possible, it must be stated (Property 1 ) if there are states of mutual allocations in digraphs $G_{W, 2}$ and $G_{S, 1}$ $\left(G_{S, 1}\right.$ - digraph including $G_{W, 1}$ and transitory path of states leading to it).

In order to determine these states, Algorithm 1 was applied. The use of Algorithm 1 depended upon the knowledge of digraph $G_{S, 1}$. It should be emphasized that the solution of the problem (10) makes it possible to determine the cyclic schedule and digraph $G_{W, 1}$ corresponding to it. It does not allow, however, determining states belonging to transitory processes. In the discussed case digraph $G_{S, 1}$ is 
TABLE 3: Times (units) of operations executions of local and multimodal processes from Figure 2.

\begin{tabular}{|c|c|c|c|c|c|c|c|c|c|c|c|c|c|}
\hline$k=1,2,3,4$ & $R_{1}$ & $R_{2}$ & $R_{3}$ & $R_{4}$ & $R_{5}$ & $R_{6}$ & $R_{7}$ & $R_{8}$ & $R_{9}$ & $R_{10}$ & $R_{11}$ & $R_{12}$ & $R_{13} \div R_{33}$ \\
\hline$m t_{1}^{k}$ & - & - & 77 & - & 64 & 77 & - & 20 & - & - & 20 & - & 1 \\
\hline$m t_{2}^{k}$ & - & 77 & - & 20 & 10 & - & 30 & - & - & 30 & - & - & 1 \\
\hline$m t_{3}^{k}$ & 77 & - & - & 49 & - & - & 20 & 50 & 20 & - & - & 10 & 1 \\
\hline$\overline{t_{1}^{1}}$ & - & - & 76 & - & - & 2 & - & - & - & - & - & - & 1 \\
\hline$t_{2}^{1}$ & - & - & - & - & 4 & 18 & - & 1 & 1 & - & - & - & 1 \\
\hline$t_{2}^{2}$ & - & - & - & - & 1 & 1 & - & 1 & 1 & - & - & - & 1 \\
\hline$t_{2}^{3}$ & - & - & - & - & 3 & 1 & - & 1 & 1 & - & - & - & 1 \\
\hline$t_{3}^{1}$ & - & - & - & - & - & - & - & - & 1 & - & - & 1 & 1 \\
\hline$t_{4}^{1}$ & - & - & - & - & - & - & 4 & 1 & - & 1 & 1 & - & 1 \\
\hline$t_{4}^{2}$ & - & - & - & - & - & - & 1 & 1 & - & 1 & 1 & - & 1 \\
\hline$t_{5}^{1}$ & 1 & 61 & - & 1 & 1 & - & - & - & - & - & - & - & 1 \\
\hline$t_{5}^{2}$ & 1 & 1 & - & 1 & 1 & - & - & - & - & - & - & - & 1 \\
\hline$t_{6}^{1}$ & - & - & - & 1 & - & - & 1 & - & - & - & - & - & 1 \\
\hline$t_{6}^{2}$ & - & - & - & 1 & - & - & 1 & - & - & - & - & - & 1 \\
\hline$t_{7}^{1}$ & - & - & - & - & - & - & 1 & - & - & 34 & - & - & 1 \\
\hline$t_{8}^{1}$ & 1 & - & - & 1 & - & - & - & - & - & - & - & - & 1 \\
\hline
\end{tabular}

TABLE 4: Times (units) of operations executions of local and multimodal processes from Figure 7(b).

\begin{tabular}{|c|c|c|c|c|c|c|c|c|c|c|c|c|c|}
\hline$k=1,2,3,4$ & $R_{1}$ & $R_{2}$ & $R_{3}$ & $R_{4}$ & $R_{5}$ & $R_{6}$ & $R_{7}$ & $R_{8}$ & $R_{9}$ & $R_{10}$ & $R_{11}$ & $R_{12}$ & $R_{13} \div R_{33}$ \\
\hline$m t_{1}^{k}$ & - & - & 91 & - & 64 & 91 & - & 20 & 20 & - & - & 20 & 1 \\
\hline$m t_{2}^{k}$ & - & 91 & - & 20 & 24 & - & 68 & 30 & - & - & 30 & - & 1 \\
\hline$m t_{3}^{k}$ & 91 & - & - & 68 & - & - & 20 & 50 & - & - & - & - & 1 \\
\hline$\overline{t_{1}^{1}}$ & - & - & 90 & - & - & 2 & - & - & - & - & - & - & 1 \\
\hline$t_{2}^{1}$ & - & - & - & - & 4 & 28 & - & 1 & 1 & - & - & - & 1 \\
\hline$t_{2}^{2}$ & - & - & - & - & 1 & 1 & - & 1 & 1 & - & - & - & 1 \\
\hline$t_{2}^{3}$ & - & - & - & - & 1 & 1 & - & 18 & 1 & - & - & - & 1 \\
\hline$t_{3}^{1}$ & - & - & - & - & - & - & - & - & 1 & - & - & 15 & 1 \\
\hline$t_{4}^{1}$ & - & - & - & - & - & - & 1 & 1 & - & 1 & 1 & - & 1 \\
\hline$t_{4}^{2}$ & - & - & - & - & - & - & 60 & 1 & - & 1 & 1 & - & 1 \\
\hline$t_{5}^{1}$ & 1 & 62 & - & 1 & 1 & - & - & - & - & - & - & - & 1 \\
\hline$t_{5}^{2}$ & 1 & 1 & - & 6 & 1 & - & - & - & - & - & - & - & 1 \\
\hline$t_{6}^{1}$ & - & - & - & 13 & - & - & 1 & - & - & - & - & - & 1 \\
\hline$t_{6}^{2}$ & - & - & - & 66 & - & - & 1 & - & - & - & - & - & 1 \\
\hline$t_{7}^{1}$ & - & - & - & - & - & - & 1 & - & - & 81 & - & - & 1 \\
\hline$t_{8}^{1}$ & 1 & - & - & 1 & - & - & - & - & - & - & - & - & 1 \\
\hline
\end{tabular}

unknown. Yet, the state of cyclic processes can be determined from digraph $G_{W, 1}$ by means of searching for parents of the states $V_{W, 1}$ not belonging to this set. Therefore, next state function $\delta$ defined in [16] was applied.

Based on the determined states, a search for mutual allocation states was carried out. An example of such states are the states $S^{19}, S^{40}$. A change of cyclic steady states based on these states (consisting of changing priority rules, semaphores and indexes) was illustrated in Figures 11 and 12.

As shown in Figure 12 the considered cyclic steady states are mutually reachable $\left(G_{W, 1} \rightarrow G_{W, 2}\right.$ and $\left.G_{W, 2} \rightarrow G_{W, 1}\right)$. In general case, however, mutual reachability is not always guaranteed. For instance, consider SCCP composed of four cyclic processes shown in Figure 13(a). The cyclic steady state space $\mathscr{P}$ consists of two vortexes $G_{S, 1}$ and $G_{S, 2}$; see Figure 13(b). There are five different transient periods linking $G_{W, 1}$ and $G_{W, 2}$; see orange arcs. There is not, however, any transient period linking $G_{S, 2}$ and $G_{S, 1}$; that is, there are not any states shared by $G_{W, 2}$ and $G_{S, 1}$ while following Property 1. Moreover, both direct mutual reachability and indirect mutual reachability are not guaranteed in general case of a cyclic steady state space.

\section{Related Work}

Many algorithms for the scheduling and routing of AGVs have been proposed. However, most of the existing results are applicable to systems with a small number of AGVs, 
TABLE 5: The timing $x_{i, j}^{k}, m x_{i, j}^{k}$ of commencement of operations of local and multimodal processes from Figure 2.

\begin{tabular}{ccccccccccccc}
\hline & $R_{1}$ & $R_{2}$ & $R_{3}$ & $R_{4}$ & $R_{5}$ & $R_{6}$ & $R_{7}$ & $R_{8}$ & $R_{9}$ & $R_{10}$ & $R_{11}$ & $R_{12}$ \\
\hline$m x_{1}^{1}$ & - & - & -10 & - & 146 & 68 & - & 211 & - & - & 232 \\
$m x_{2}^{1}$ & - & 55 & - & 144 & 133 & - & 165 & - & - & 190 & - & - \\
$m x_{3}^{1}$ & 9 & - & - & 87 & - & - & 137 & 158 & 209 & - & - \\
\hline$x_{1}^{1}$ & - & - & -9 & - & - & 68 & - & - & - & - & - & - \\
$x_{2}^{1}$ & - & - & - & - & 66 & 47 & - & -8 & -6 & - & - & - \\
$x_{2}^{2}$ & - & - & - & - & 45 & 71 & - & 48 & 49 & - & - & - \\
$x_{2}^{3}$ & - & - & - & - & 47 & -7 & - & 51 & 70 & - & - & - \\
$x_{3}^{1}$ & - & - & - & - & - & - & - & - & 68 & - & - & -10 \\
$x_{4}^{1}$ & - & - & - & - & - & - & -7 & -2 & - & 71 & 0 \\
$x_{4}^{2}$ & - & - & - & - & - & - & -2 & 70 & - & -6 & -6 \\
$x_{5}^{1}$ & 69 & -9 & - & 57 & 53 & - & - & - & - & - & - \\
$x_{5}^{2}$ & -9 & 53 & - & 64 & 62 & - & - & - & - & - & - \\
$x_{6}^{1}$ & - & - & - & 3 & - & - & 5 & - & - & - & - \\
$x_{6}^{2}$ & - & - & - & 55 & - & - & 57 & - & - & - & - \\
$x_{7}^{1}$ & - & - & - & - & - & - & 34 & - & - & - \\
$x_{8}^{1}$ & 5 & - & - & 7 & - & - & - & - & - & - \\
\hline
\end{tabular}

TABLE 6: The timing $x_{i, j}^{k}, m x_{i, j}^{k}$ of commencement of operations of local and multimodal processes from Figure 7(b).

\begin{tabular}{cccccccccccccc}
\hline & $R_{1}$ & $R_{2}$ & $R_{3}$ & $R_{4}$ & $R_{5}$ & $R_{6}$ & $R_{7}$ & $R_{8}$ & $R_{9}$ & $R_{10}$ & $R_{11}$ & $R_{12}$ \\
\hline$m x_{1}^{1}$ & - & - & -10 & - & 174 & 82 & - & 239 & 260 & - & - \\
$m x_{2}^{1}$ & - & 55 & - & 172 & 147 & - & 193 & 262 & - & - & 293 \\
$m x_{3}^{1}$ & 9 & - & - & 101 & - & - & 170 & - & - & 191 & - \\
\hline$x_{1}^{1}$ & - & - & -9 & - & - & 82 & - & - & - & - & - \\
$x_{2}^{1}$ & - & - & - & - & 80 & 51 & - & -9 & -1 & - & - & - \\
$x_{2}^{2}$ & - & - & - & - & 49 & 85 & - & 51 & 53 & - & - & - \\
$x_{2}^{3}$ & - & - & - & - & 51 & -7 & - & 53 & 72 & - & - \\
$x_{3}^{1}$ & - & - & - & - & - & - & - & - & 91 & - & - \\
$x_{4}^{1}$ & - & - & - & - & - & - & -7 & -5 & - & 85 & -1 \\
$x_{4}^{2}$ & - & - & - & - & - & - & 13 & 74 & - & 11 & 103 \\
$x_{5}^{1}$ & 78 & -10 & - & 76 & 53 & - & - & - & - & - & - \\
$x_{5}^{2}$ & -9 & 53 & - & 78 & 76 & - & - & - & - & - & - \\
$x_{6}^{1}$ & - & - & - & -9 & - & - & 5 & - & - & - & - \\
$x_{6}^{2}$ & - & - & - & 9 & - & - & 76 & - & - & - & - \\
$x_{7}^{1}$ & - & - & - & - & - & - & 1 & - & - & - \\
$x_{8}^{1}$ & 5 & - & - & 7 & - & - & - & - & - & - \\
\hline
\end{tabular}

offering a low degree of concurrency. With a drastically increased number of AGVs in recent applications (e.g., in the order of a hundred in a container handling system), the combinatorial nature of the fleet scheduling and assignment problems rapidly increases the complexity. For instance, it appears clearly through constraints which relate to assigning a fleet to a route. Suppose that there are $n$ fleet and $m$ routes, the number of binary decisions variables is $m \times n$ and theoretically, there are $2^{n \times m}$ solutions to be considered. In fact, according to computational complexity, this problem is NP-hard [2]. A number of papers are concerned with the fleet assignment problem $[1-3,8]$ and maintenance planning $[6,7$, 21]. However, few papers have considered the combination of fleet assignment and maintenance planning [2, 22]. Most publications on fleet assignment have been focused on the assignment of fleets to minimize the fleet operation cost $[1,3$, 8 ]. Some papers on maintenance planning have been focused on planning maintenance tasks to minimize the maintenance cost $[6,7,21]$. Few papers have considered the integration of fleet assignment and maintenance planning [2, 22]. The inventory policy with preventive maintenance consideration is mostly studied for production system $[6,23,24]$. Because of unpredicted situations, rescheduling is required to deal with online decision making. Finally, scheduling and rescheduling fleet assignment and maintenance planning and inventory policy are implemented as a decision support system [22].

Because most of real-life available manufacturing processes manifest their cyclic steady state the alternative 


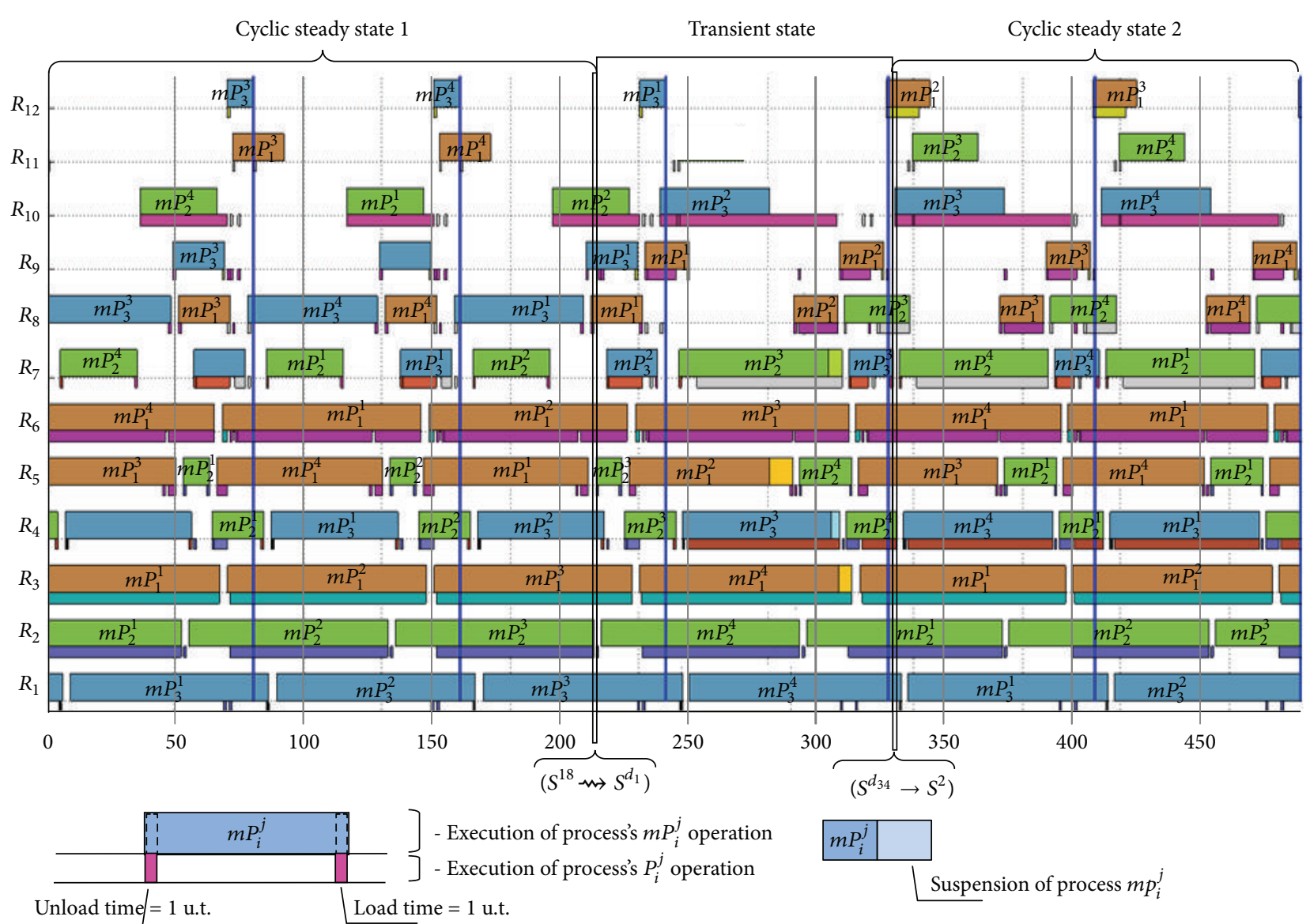

FIGURE 10: The indirect transition between schedules from Figures 4 and 6.

approach to AGVs fleet dispatching and scheduling, for example, following from the SCCP concept $[1,9,25]$ can be considered as well. Many models and methods have been proposed to solve the cyclic scheduling problem so far. Most of these are oriented at finding a minimal cycle or maximal throughput while assuming deadlock-free processes flow. The approaches trying to estimate the cycle time from cyclic processes structure and the synchronization mechanism employed (i.e., mutual exclusion instances) are quite unique.

In that context our main contribution is to propose a new modeling framework enabling to state and answer the following questions: Does there exist a control procedure (e.g., a set of dispatching rules and an initial state) guaranteeing an assumed steady cyclic state subject to SCCP's structure constraints? Does an SCCP's structure exist such that a steady cyclic state can be achieved?

Is the cyclic steady sate space empty? In the case the space is not empty the next question is do the cyclic steady states are mutually reachable?

The last question refers to the problem of multimodal processes rescheduling. Most of so far proposed approaches to the rescheduling of processes executed within multimodal transportation networks environment $[17,26-28]$ are only limited to the deadlock-free cases. It should be noted that, however, the evolutionary algorithms driven approaches frequently used [26-28] are not effective in cases allowing deadlocks occurrence. The approach proposed allows finding deadlock-free transitions between the reachable cyclic steady states while avoiding the deadlocks in the course of the transient state generation.

\section{Conclusions}

The complicated problem which integrates AGVs fleet assignment, routing, and scheduling, generating a suboptimal cyclic schedule for multiproduct manufacturing, is novelty of this research. Such integrated AGVs dispatching problems can be seen as a special case of the cyclic blocking flowshop one, where the jobs might block either the machine or the AGV at the processing time. Therefore the main class of problems of AGVs fleet match-up scheduling with reference schedule of assumed production flow belong to the NP-hard ones.

Besides the above mentioned AGVs dispatching/planning issues the research objective regards a quite large class of digital and/or logistics networks that share common properties even though they have huge intrinsic differences. In other words, besides AGVs that can be treated as a kind of internal transport, other emerging trends concerning the logistics (e.g., supply chains management) and city traffic 

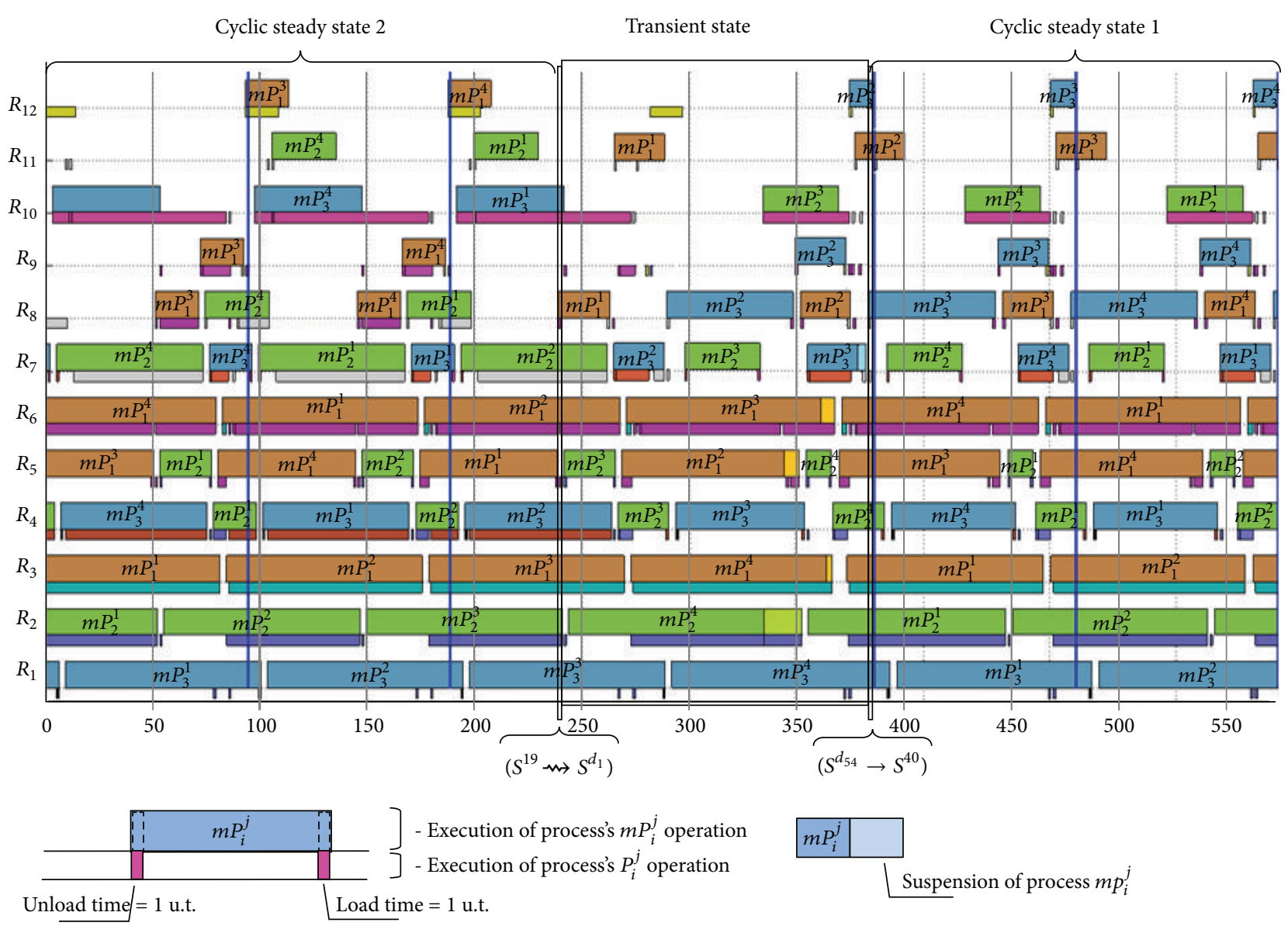

FIgURE 11: The indirect transition between schedules from Figures 6 and 4.

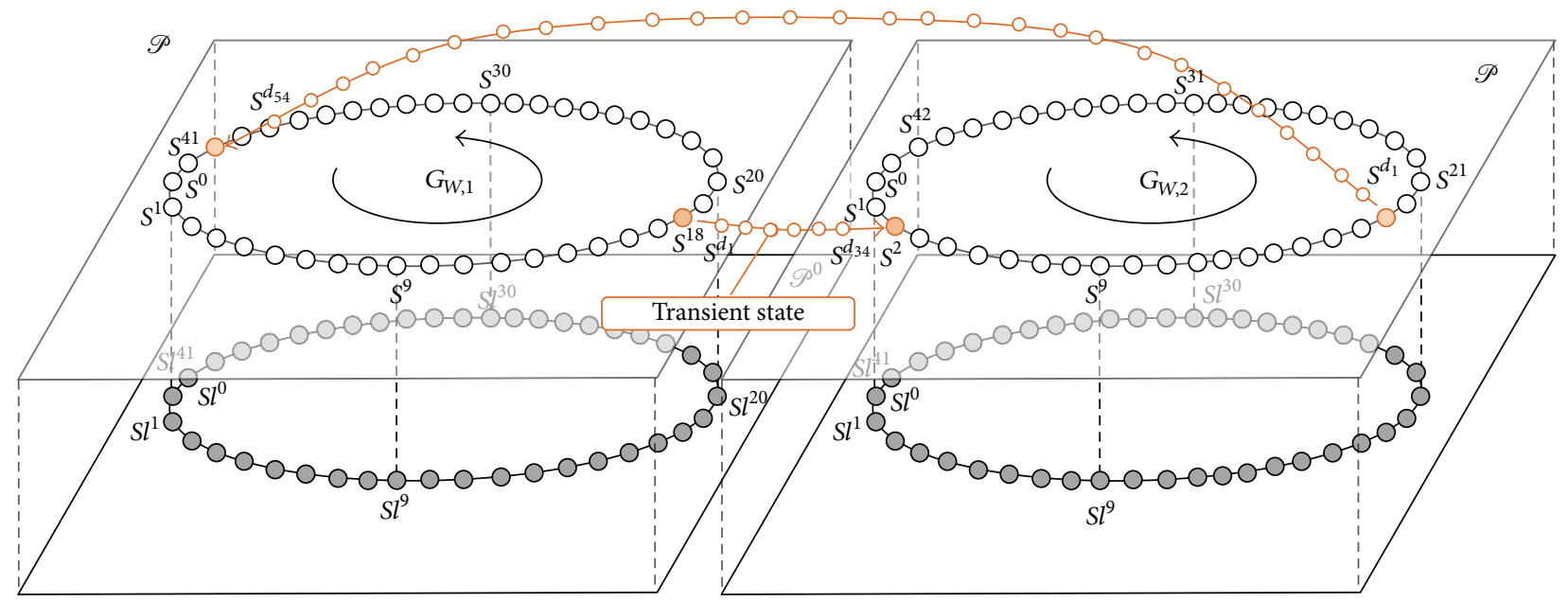

FIgURE 12: The indirect transition between cyclic steady states: $G_{W, 1} \rightarrow G_{W, 2}$ and $G_{W, 2} \rightarrow G_{W, 1}$.

(e.g., infrastructure of the public transport) issues can be modeled. For instance, the relevant multimodal processes of passengers traveling between destination points in an environment of local processes encompassing the subway lines network might be considered as well. In other words, the passenger's itinerary including different metro lines can be considered as a plan of a multimodal process routing within a metro network.

The proposed declarative approach aimed at AGVs fleet scheduling stated in terms of constraint satisfaction problem 


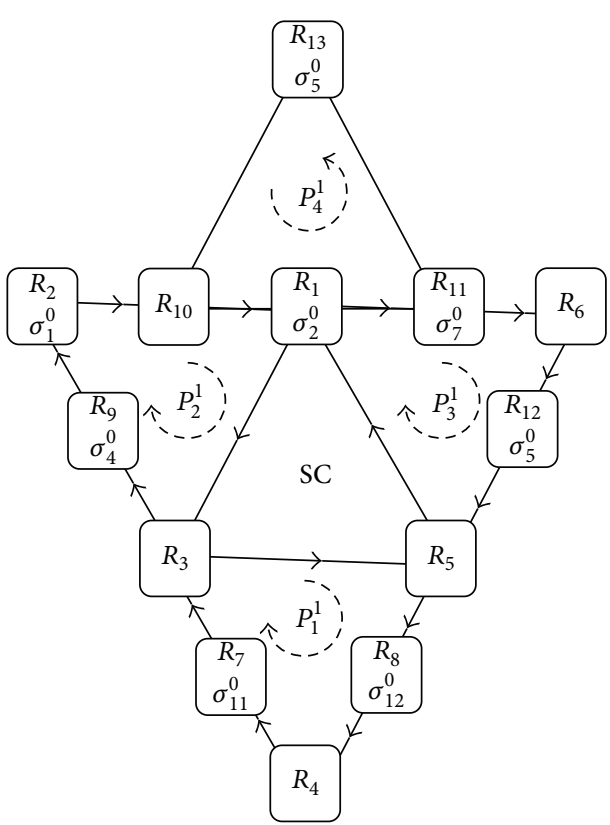

(a)

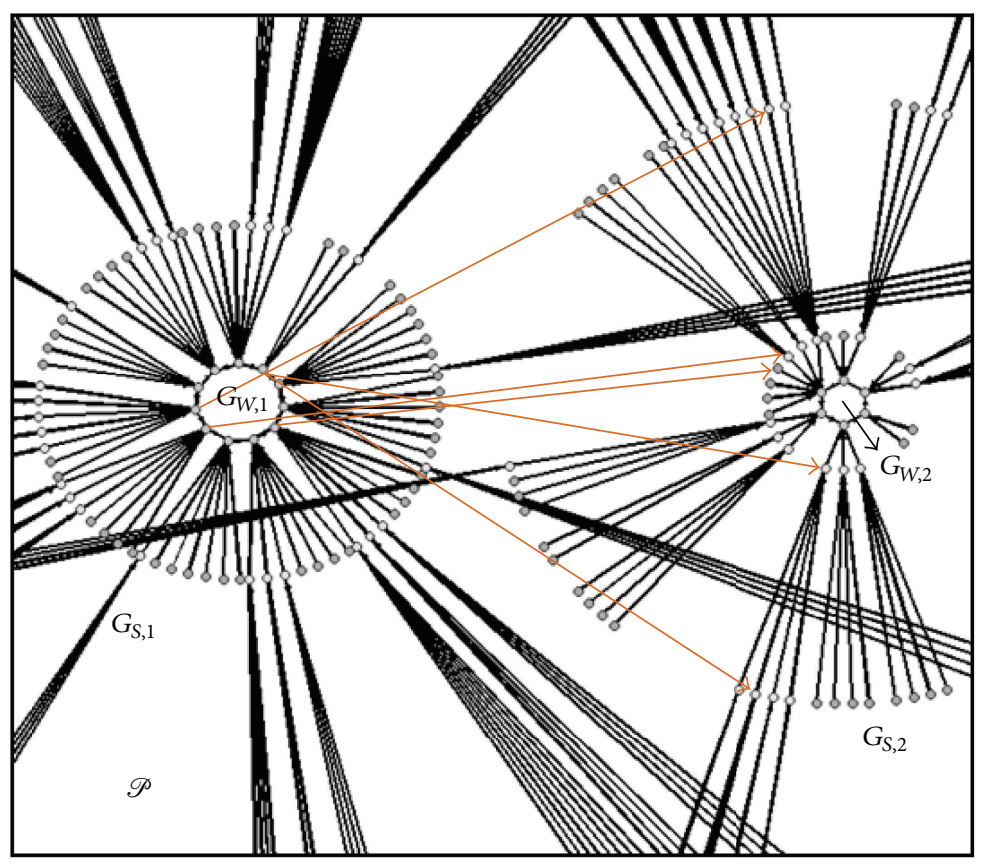

(b)

FIGURE 13: Illustration of SCCP composed of four local cyclic processes (a) and cyclic steady state space $\mathscr{P}$ including transient states depicting mutual reachability $G_{W, 1} \rightarrow G_{W, 2}(\mathrm{~b})$.

representation provides a unified method for performance evaluation of local as well as supported by them multimodal processes.

In general case, however, there is not any guarantee that the considered SCCP model of AGVS has a nonempty cyclic steady states space as well as that in case this space is not empty, any direct or indirect mutual reachability can be observed. That means that the decidability of the problem of the space $\mathscr{P}$ reachability and mutual reachability among its cyclic steady states plays a pivotal role in multimodal processes rescheduling.

\section{References}

[1] J. Abara, "Applying integer linear programming to the fleet assignment problem," Interfaces, vol. 19, pp. 4-20, 1989.

[2] L. W. Clarke, C. A. Hane, E. L. Johnson, and G. L. Nemhauser, "Maintenance and crew considerations in fleet assignment," Transportation Science, vol. 30, no. 3, pp. 249-260, 1996.

[3] M. D. D. Clarke, "Irregular airline operations: a review of the state-of-the-practice in airline operations control centers," Journal of Air Transport Management, vol. 4, no. 2, pp. 67-76, 1998.

[4] N. G. Hall, C. Sriskandarajah, and T. Ganesharajah, "Operational decisions in AGV-served flowshop loops: fleet sizing and decomposition," Annals of Operations Research, vol. 107, no. 1-4, pp. 189-209, 2001.

[5] S. Hoshino and H. Seki, "Multi-robot coordination for jams in congested systems," Robotics and Autonomous Systems, vol. 61, no. 8, pp. 808-820, 2013.
[6] A. Chelbi and D. Ait-Kadi, "Analysis of a production/inventory system with randomly failing production unit submitted to regular preventive maintenance," European Journal of Operational Research, vol. 156, no. 3, pp. 712-718, 2004.

[7] S. Deris, S. Omatu, H. Ohta, L. C. Shaharudin Kutar, and P. Abd Samat, "Ship maintenance scheduling by genetic algorithm and constraint-based reasoning," European Journal of Operational Research, vol. 112, no. 3, pp. 489-502, 1999.

[8] C. A. Hane, C. Barnhart, E. L. Johnson, R. E. Marsten, G. L. Nemhauser, and G. Sigismondi, "The fleet assignment problem: solving a large-scale integer program," Mathematical Programming, vol. 70, no. 2, pp. 211-232, 1995.

[9] L. Qiu, W.-J. Hsu, S.-Y. Huang, and H. Wang, "Scheduling and routing algorithms for AGVs: a survey," International Journal of Production Research, vol. 40, no. 3, pp. 745-760, 2002.

[10] B. Trouillet, O. Korbaa, and J.-C. Gentina, "Formal approach of FMS cyclic scheduling," IEEE Transactions on Systems, Man and Cybernetics C, vol. 37, no. 1, pp. 126-137, 2007.

[11] E. Levner, V. Kats, D. Alcaide López De Pablo, and T. C. E. Cheng, "Complexity of cyclic scheduling problems: a state-ofthe-art survey," Computers and Industrial Engineering, vol. 59, no. 2, pp. 352-361, 2010.

[12] T. Von Kampmeyer, Cyclic scheduling problems [Ph.D. dissertation], Fachbereich Mathematik/Informatik, Universität Osnabrück, 2006.

[13] M. Polak, P. B. Z. Majdzik, and R. Wójcik, "The performance evaluation tool for automated prototyping of concurrent cyclic processes," Fundamenta Informaticae, vol. 60, no. 1-4, pp. 269289, 2004.

[14] G. Bocewicz and Z. Banaszak, "Declarative approach to cyclic scheduling of multimodal processes," in EcoProduction and 
Logistics, P. Golińska, Ed., pp. 203-238, Springer, Heidelberg, Germany, 2013.

[15] G. Bocewicz and Z. Banaszak, "Declarative approach to cyclic steady states space refinement: periodic processes scheduling," International Journal of Advanced Manufacturing Technology, vol. 67, no. 1-4, pp. 137-155, 2013.

[16] G. Bocewicz, R. Wójcik, and Z. Banaszak, "Cyclic Steady State Refinement," in Proceedings of the International Symposium on Distributed Computing and Artificial Intelligence, A. Abraham, J. M. Corchado, S. Rodríguez González, and J. F. de Paz Santana, Eds., vol. 91 of Advances in Intelligent and Soft Computing, pp. 191-198, Springer, Berlin, Germany, 2011.

[17] M. Dridi, K. Mesghouni, and P. Borne, "Traffic control in transportation systems," Journal of Manufacturing Technology Management, vol. 16, no. 1, pp. 53-74, 2005.

[18] J.-S. Song and T.-E. Lee, "Petri net modeling and scheduling for cyclic job shops with blocking," Computers and Industrial Engineering, vol. 34, no. 2-4, pp. 281-295, 1998.

[19] A. Che and C. Chu, "Optimal scheduling of material handling devices in a PCB production line: problem formulation and a polynomial algorithm," Mathematical Problems in Engineering, vol. 2008, Article ID 364279, 21 pages, 2008.

[20] G. Bocewicz, I. Nielsen, and Z. Banaszak, "AGVsfleet match-up scheduling with production flow considerations," Engineering Applications of Artificial Intelligence. In press.

[21] N. Papakostas, P. Papachatzakis, V. Xanthakis, D. Mourtzis, and G. Chryssolouris, "An approach to operational aircraft maintenance planning," Decision Support Systems, vol. 48, no. 4, pp. 604-612, 2010.

[22] W. El Moudani and F. Mora-Camino, "A dynamic approach for aircraft assignment and maintenance scheduling by airlines," Journal of Air Transport Management, vol. 6, no. 4, pp. 233-237, 2000.

[23] N. Rezg, S. Dellagi, and A. Chelbi, "Joint optimal inventory control and preventive maintenance policy," International Journal of Production Research, vol. 46, no. 19, pp. 5349-5365, 2008.

[24] T. S. Vaughan, "Failure replacement and preventive maintenance spare parts ordering policy," European Journal of Operational Research, vol. 161, no. 1, pp. 183-190, 2005.

[25] M. Sharma, "Control classification of automated guided vehicle systems," International Journal of Engineering and Advanced Technology, vol. 2, no. 1, pp. 191-196, 2012.

[26] B. F. Chaar and S. Hammadi, "Evolutionary approach to the regulation of the traffic of a system of multimodal transport," Journal Europeen des Systemes Automatises, vol. 38, no. 7-8, pp. 901-931, 2004.

[27] A. K.-S. Wong, Optimization of container process at multimodal container terminals [Ph.D. thesis], Queensland University of Technology, Brisbane, Australia, 2008.

[28] S. Zidi and S. Maouche, "Ant colony optimization for the rescheduling of multimodal transport networks," in Proceedings of the IMACS Multiconference on Computational Engineering in Systems Applications, vol. 1, pp. 965-9971, 2006. 


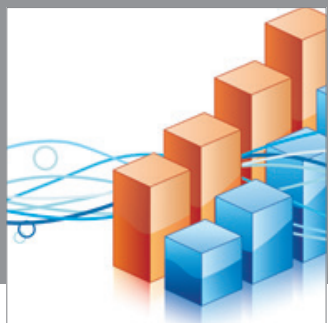

Advances in

Operations Research

mansans

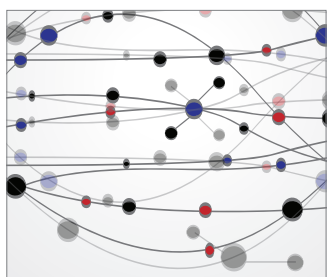

The Scientific World Journal
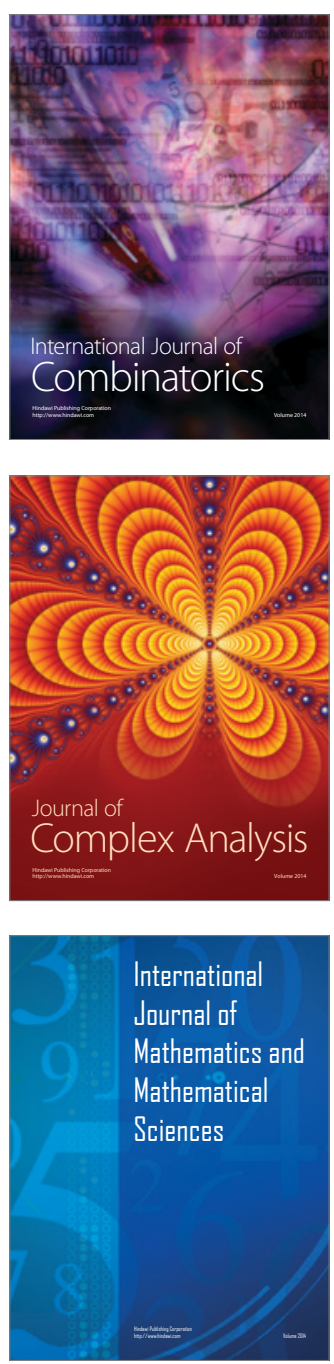
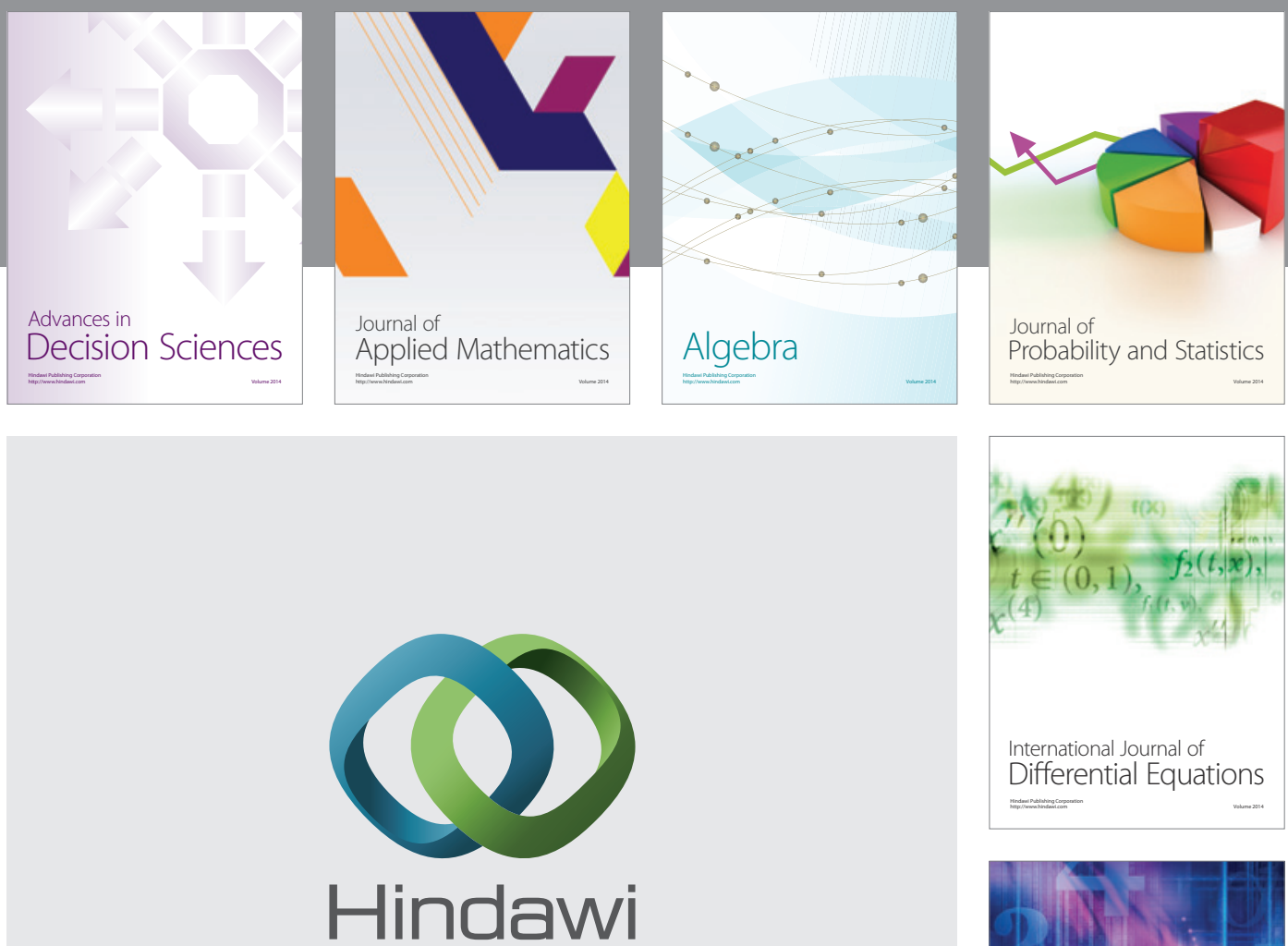

Submit your manuscripts at http://www.hindawi.com
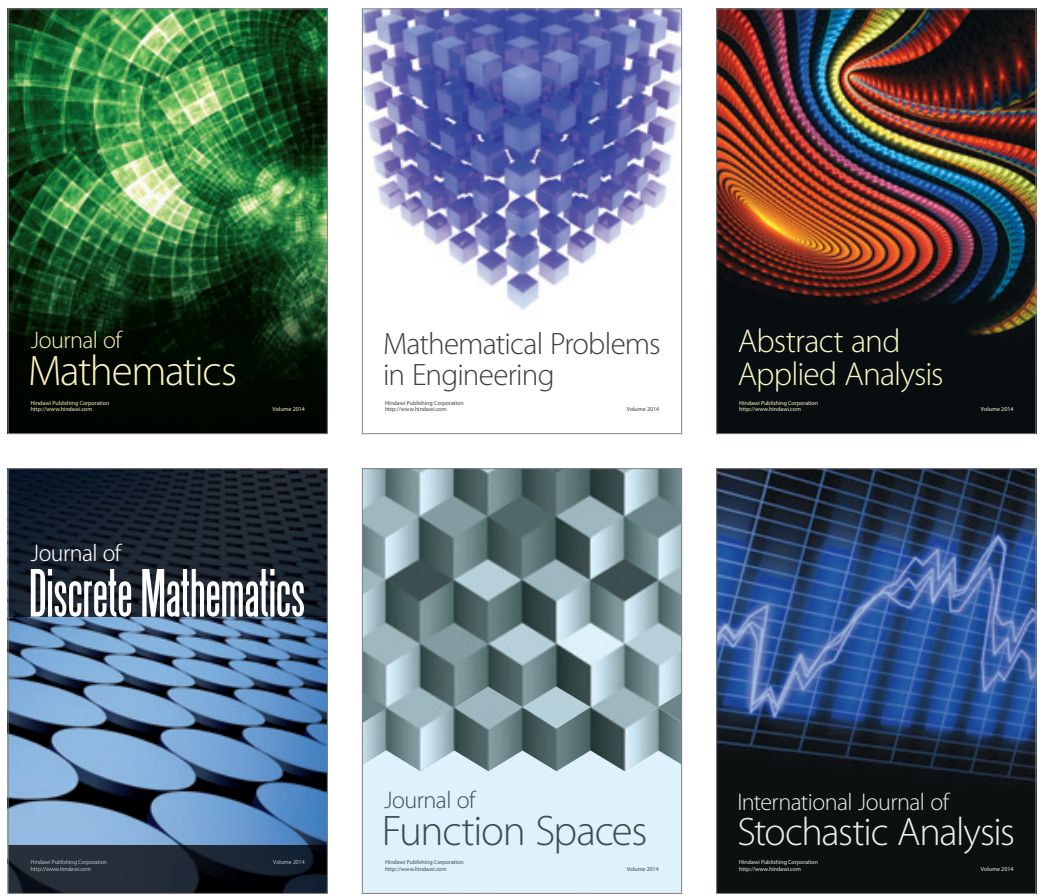

Journal of

Function Spaces

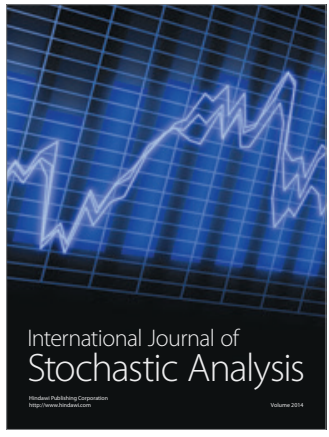

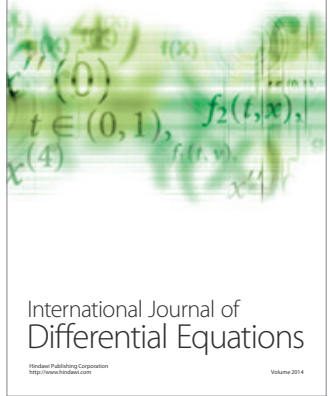
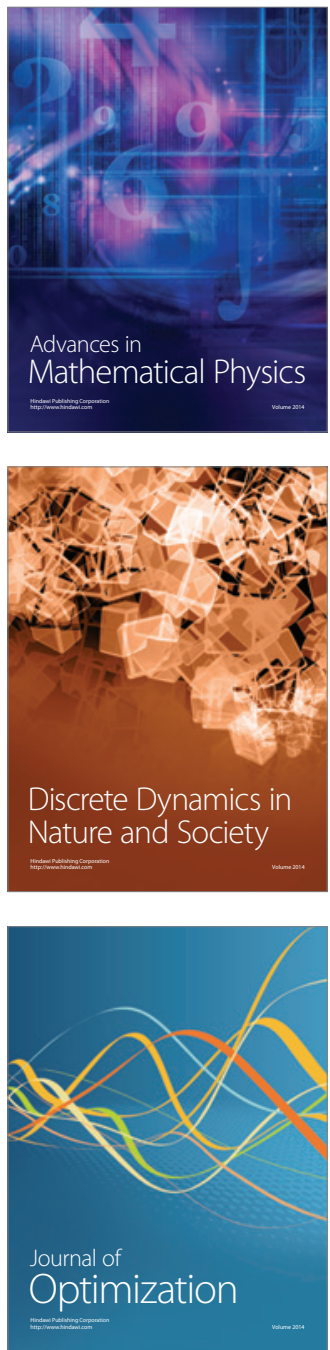